\title{
Multigrid Discretization and Iterative Algorithm for Mixed Variational Formulation of the Eigenvalue Problem of Electric Field
}

\author{
Yidu Yang, Yu Zhang, and Hai Bi \\ School of Mathematics and Computer Science, Guizhou Normal University, Guiyang 550001, China \\ Correspondence should be addressed to Yidu Yang, ydyang@gznu.edu.cn
}

Received 9 July 2012; Revised 4 September 2012; Accepted 12 September 2012

Academic Editor: Xinan Hao

Copyright (C) 2012 Yidu Yang et al. This is an open access article distributed under the Creative Commons Attribution License, which permits unrestricted use, distribution, and reproduction in any medium, provided the original work is properly cited.

This paper discusses highly finite element algorithms for the eigenvalue problem of electric field. Combining the mixed finite element method with the Rayleigh quotient iteration method, a new multi-grid discretization scheme and an adaptive algorithm are proposed and applied to the eigenvalue problem of electric field. Theoretical analysis and numerical results show that the computational schemes established in the paper have high efficiency.

\section{Introduction}

The finite element method for eigenvalue problem of electric field has become a hot topic in the field of mathematics and physics (see, e.g., [1-7]). This paper discusses high efficient mixed finite element calculation schemes for the eigenvalue problem of electric field.

Kikuchi [6] introduced the first type of mixed variational formulation for the eigenvalue problem of electric field. Based on this formulation, in [3] Buffa et al.analyzed the approximation of nodal finite element. Boffi et al. [1] discussed the second type of mixed variational formulation for the eigenvalue problem of electric field and analyzed approximations of edge element and nodal element. Yang et al. [7] studied a two-grid discretization scheme of finite element for the first type of mixed variational formulation.

Based on the work mentioned above, in this paper a new multi-grid discretization scheme and an adaptive algorithm are proposed for the first type of mixed variational formulation of eigenvalue problem and applied to the eigenvalue problem of electric field. The main features of the research in this paper are as follows. 
(1) Our multi-grid discretization scheme and adaptive algorithm, which are the extension of conforming finite element multi-grid discretization scheme (see scheme 3 in [8] and scheme 1 in [9]), are a combination of the mixed finite element method and the Rayleigh quotient iteration method (see the algorithm 27.3 in [10]). With our algorithm one solves an eigenvalue problem on a coarse grid just at the first step and always solves a linear algebraic system on finer and finer grids at each following step. We derive the error estimates for the algorithm and prove that the constants appeared in the error estimates are independent of the iteration degrees. Thus we prove the convergence of iterations.

(2) The eigenvalue problem of electric field is so complicated that it is very difficult to obtain local a posteriori error estimates for the eigenfunctions of mixed finite element. As yet, there is no achievement reported in this field. Our adaptive algorithm substitutes the weight method established by Costabel and Dauge (see $[3,11])$ for local refinement, which uses $\theta \times\left|\lambda^{h_{l}}-\lambda^{h_{l-1}}\right|(\theta \in(0,1])$ as a posteriori error estimator of $\lambda^{h_{l}}$ instead of estimating local a posteriori error for the eigenfunction. And the results are satisfying.

(3) We analyze the mixed finite element error for the eigenvalue problem of electric field (see Theorem 2.2 and Theorem 4.2). We refer to [12] to propose a new proof method which differs from the usual one in [13].

The rest of this paper is organized as follows. Some preliminaries of finite element approximations for eigenvalue problems which are needed in this paper are provided in the next section. In Section 3, for the first type of mixed variational formulation of eigenvalue problem, the finite element multi-grid discretization scheme and the adaptive algorithm are established and the validity of these schemes is proved theoretically. In Section 4, the multi-grid discretization scheme is applied to the eigenvalue problem of electric field. Finally, numerical experiments are presented in Section 5.

\section{Preliminaries}

Let $V, W$, and $D$ be three real Hilbert spaces with inner products and norms $(\cdot, \cdot)_{V},\|\cdot\|_{V}$, $(\cdot, \cdot)_{W},\|\cdot\|_{W},(\cdot, \cdot)_{D}$, and $\|\cdot\|_{D}$, respectively. Suppose that $V \hookrightarrow D$ (continuously imbedded), $a(\cdot, \cdot)$ is a symmetric, continuous, and $V$-elliptic bilinear form on $V \times V$, that is,

$$
\begin{gathered}
|a(q, \psi)| \leq M_{1}\|q\|_{V}\|\psi\|_{V}, \quad \forall q, \psi \in V, \\
a(q, q) \geq v\|q\|_{V^{\prime}}^{2}, \quad \forall 0 \neq q \in V .
\end{gathered}
$$

$b(\cdot, \cdot)$ is a continuous bilinear form on $V \times W$, that is,

$$
|b(\psi, v)| \leq M_{2}\|\psi\|_{V}\|v\|_{W}, \quad \forall \psi \in V, v \in W
$$

It is obvious that $a(\cdot, \cdot)$ is an inner product on $V$ and $\|\cdot\|_{a}=\sqrt{a(\cdot, \cdot)}$ is an equivalent norm to $\|\cdot\|_{V}$. 
In scientific and engineering computations, many eigenvalue problems have the following first type of mixed variational formulation: find $(\lambda, u, \sigma) \in R \times V \times W,(u, \sigma) \neq(0,0)$, such that

$$
\begin{gathered}
a(u, \psi)+b(\psi, \sigma)=\lambda(u, \psi)_{D^{\prime}} \quad \forall \psi \in V, \\
b(u, v)=0, \quad \forall v \in W .
\end{gathered}
$$

In order to solve problem (2.3)-(2.4), one should construct finite element spaces $V_{h} \subset V$ and $W_{h} \subset W$. Restricting (2.3)-(2.4) on $V_{h} \times W_{h}$, we get the conforming mixed finite element approximation as follows: find $\left(\lambda_{h}, u_{h}, \sigma_{h}\right) \in R \times V_{h} \times W_{h},\left(u_{h}, \sigma_{h}\right) \neq(0,0)$, such that

$$
\begin{gathered}
a\left(u_{h}, \psi\right)+b\left(\psi, \sigma_{h}\right)=\lambda_{h}\left(u_{h}, \psi\right)_{D^{\prime}} \quad \forall \psi \in V_{h}, \\
b\left(u_{h}, v\right)=0, \quad \forall v \in W_{h} .
\end{gathered}
$$

Consider the associated source and approximate source problems. Given $f \in D$, find $(w, p) \in V \times W$ satisfying

$$
\begin{gathered}
a(w, \psi)+b(\psi, p)=(f, \psi)_{D^{\prime}}, \quad \forall \psi \in V, \\
b(w, v)=0, \quad \forall v \in W .
\end{gathered}
$$

Given $f \in D$, find $\left(w_{h}, p_{h}\right) \in V_{h} \times W_{h}$ satisfying

$$
\begin{gathered}
a\left(w_{h}, \psi\right)+b\left(\psi, p_{h}\right)=(f, \psi)_{D^{\prime}} \quad \forall \psi \in V_{h}, \\
b\left(w_{h}, v\right)=0, \quad \forall v \in W_{h} .
\end{gathered}
$$

Note that the source term $f$ is independent of the solution.

As for the mixed finite element method for boundary value problems, Brezzi and Fortinand so forth have established a systematic theory (see [14]). By Brezzi-Babuska Theorem, we have the following.

Lemma 2.1 (Brezzi-Babuska). Suppose that

(C1) (2.1)-(2.2) hold;

(C2) inf-sup condition holds, namely, there exists a constant $v_{1}>0$, such that

$$
\sup _{\psi \in V, \psi \neq 0} \frac{b(\psi, v)}{\|\psi\|_{V}} \geq v_{1}\|v\|_{W}, \quad \forall v \in W
$$

then there exists a unique solution $(w, p)$ to the problem (2.7) and

$$
\|w\|_{a}+\|p\|_{W} \leq C_{r}\|f\|_{D^{\prime}}
$$

where $C_{r}$ just depends on $v, v_{1}$, and $M_{1}$. Moreover, suppose; 
(C3) discrete inf-sup condition holds, namely, there exists a constant $v_{2}>0$ independent of $h$, such that

$$
\sup _{\psi \in V_{h}, \psi \neq 0} \frac{b(\psi, v)}{\|\psi\|_{V}} \geq v_{2}\|v\|_{W}, \quad \forall v \in W_{h}
$$

then there exists a unique solution $\left(w_{h}, p_{h}\right)$ to the problem (2.8) and the following error estimate is valid:

$$
\left\|w-w_{h}\right\|_{a}+\left\|p-p_{h}\right\|_{W} \leq C_{e}\left\{\inf _{q \in V_{h}}\|w-q\|_{a}+\inf _{v \in W_{h}}\|p-v\|_{W}\right\}
$$

where $C_{e}$ just depends on $v, v_{2}$ and $M_{1}, M_{2}$.

Suppose conditions (C1)-(C3) hold in Lemma 2.1. Then there exist unique solutions to the problem (2.7) and (2.8), respectively. Thus, we can define linear bounded operators as follows: $T: D \rightarrow V, S: D \rightarrow W: \forall f \in D$,

$$
\begin{gathered}
a(T f, \psi)+b(\psi, S f)=(f, \psi)_{D^{\prime}} \quad \forall \psi \in V, \\
b(T f, v)=0, \quad \forall v \in W .
\end{gathered}
$$

$T_{h}: D \rightarrow V_{h} \subset V, S_{h}: D \rightarrow W_{h} \subset W: \forall f \in D$,

$$
\begin{gathered}
a\left(T_{h} f, \psi\right)+b\left(\psi, S_{h} f\right)=(f, \psi)_{D^{\prime}}, \quad \forall \psi \in V_{h} ; \\
b\left(T_{h} f, v\right)=0, \quad \forall v \in W_{h} .
\end{gathered}
$$

Obviously, (2.3)-(2.4) has the following equivalent operator form

$$
\begin{gathered}
\lambda T u=u, \\
\sigma=S(\lambda u),
\end{gathered}
$$

and (2.5)-(2.6) has the following equivalent operator form

$$
\begin{gathered}
\lambda_{h} T_{h} u_{h}=u_{h}, \\
\sigma_{h}=S_{h}\left(\lambda_{h} u_{h}\right) .
\end{gathered}
$$

It is easy to verify that $T: D \rightarrow D, T_{h}: D \rightarrow D$ are self-adjoint operators in the sense of inner product $(\cdot, \cdot)_{D}$ (see [7]). 
Assume $V \stackrel{c}{\hookrightarrow} D$ (compactly embedded), then it's easy to prove that $T: D \rightarrow D$ is completely continuous, $T: V \rightarrow V$ is completely continuous, and $T_{h}$ is a finite rank operator. Combining (2.3)-(2.4), (2.5)-(2.6), and the $V$-ellipticity of $a(\cdot, \cdot)$, we deduce

$$
\lambda=\frac{a(u, u)}{(u, u)_{D}}>0, \quad \lambda_{h}=\frac{a\left(u_{h}, u_{h}\right)}{\left(u_{h}, u_{h}\right)_{D}}>0
$$

Then from the spectral theory of self-adjoint and completely continuous operator, we know that the eigenvalues of (2.3)-(2.4) can be sorted as

$$
0<\lambda_{1} \leq \lambda_{2} \leq \cdots \leq \lambda_{k} \leq \cdots \nearrow+\infty
$$

and the corresponding eigenfunctions are

$$
\left(u_{1}, \sigma_{1}\right),\left(u_{2}, \sigma_{2}\right), \ldots,\left(u_{k}, \sigma_{k}\right), \ldots,
$$

where $\left(u_{i}, u_{j}\right)_{D}=\delta_{i j}$.

The eigenvalues of (2.5)-(2.6) can be sorted as

$$
0<\lambda_{1, h} \leq \lambda_{2, h} \leq \cdots \leq \lambda_{k, h} \leq \cdots \leq \lambda_{N_{h}, h}
$$

and the corresponding eigenfunctions are

$$
\left(u_{1, h}, \sigma_{1, h}\right),\left(u_{2, h}, \sigma_{2, h}\right), \ldots,\left(u_{k, h}, \sigma_{k, h}\right), \ldots,\left(u_{N_{h}, h}, \sigma_{N_{h}, h}\right)
$$

where $N_{h}=\operatorname{dim} V_{h},\left(u_{i, h}, u_{j, h}\right)_{D}=\delta_{i j}$.

From (2.5), by taking $u_{h}=u_{i, h}, \psi=u_{j, h}$, we have

$$
a\left(u_{i, h}, u_{j, h}\right)+b\left(u_{j, h}, \sigma_{h}\right)=\lambda_{i, h}\left(u_{i, h}, u_{j, h}\right)_{D^{\prime}}
$$

and from (2.6) we see that $b\left(u_{j, h}, \sigma_{h}\right)=0$, then

$$
\mathrm{a}\left(u_{i, h}, u_{j, h}\right)=\lambda_{i, h}\left(u_{i, h}, u_{j, h}\right)_{D}=\lambda_{i, h} \delta_{i j}
$$

thus $\left\{u_{i, h} /\left\|u_{i, h}\right\|_{a}\right\}$ is a completely normal eigenvector system on $V_{h}$ in the sense of the inner product $a(\cdot, \cdot)$.

Denote $\lambda_{k}=1 / \mu_{k}, \lambda_{k, h}=1 / \mu_{k, h}$. In this paper, $\mu_{k}$ and $\mu_{k, h}, \lambda_{k}$ and $\lambda_{k, h}$ are all called eigenvalues.

Let $\mu$ be the $k$ th eigenvalue with algebraic multiplicity $q, \mu=\mu_{k}=\mu_{k+1}=\cdots=\mu_{k+q-1}$. $M(\mu)$ is the space spanned by all eigenfunctions $\left\{u_{j}\right\}_{k}^{k+q-1}$ corresponding to $\mu$ of $T . M_{h}(\mu)$ is the space spanned by all eigenfunctions $\left\{u_{j, h}\right\}_{k}^{k+q-1}$ corresponding to all eigenvalues of $T_{h}$ that converge to $\mu$. Let $\widehat{M}(\mu)=\left\{v: v \in M(\mu),\|v\|_{a}=1\right\}, \widehat{M}_{h}(\mu)=\left\{v: v \in M_{h}(\mu),\|v\|_{a}=1\right\}$. 
We call $\lambda=1 / \mu$ as the $k$ th eigenvalue, too. Denote $M(\lambda)=M(\mu), M_{h}(\lambda)=M_{h}(\mu)$, and $\widehat{M}(\lambda)=\widehat{M}(\mu)$. Define

$$
\begin{aligned}
& \left\|\left.\left(T-T_{h}\right)\right|_{M(\lambda)}\right\|_{D}=\max _{u \in M(\lambda)} \frac{\left\|\left(T-T_{h}\right) u\right\|_{D}}{\|u\|_{D}}, \\
& \left\|\left.\left(T-T_{h}\right)\right|_{M(\lambda)}\right\|_{a}=\max _{u \in M(\lambda)} \frac{\left\|\left(T-T_{h}\right) u\right\|_{a}}{\|u\|_{a}} .
\end{aligned}
$$

The convergence and error estimates about the mixed element method of eigenvalue problem have been studied in $[7,12,13,15,16]$. From [12], we know that the following results are valid.

Theorem 2.2. Suppose $V \stackrel{c}{\hookrightarrow} D, a(u, v)$ is symmetric, and the conditions of Lemma 2.1 hold; moreover, for any $f \in D$,

$$
\begin{aligned}
& \inf _{q \in V_{h}}\|T f-q\|_{V} \longrightarrow 0 \quad(h \longrightarrow 0), \\
& \inf _{v \in W_{h}}\|S f-v\|_{W} \longrightarrow 0 \quad(h \longrightarrow 0) .
\end{aligned}
$$

Then $\left\|T_{h}-T\right\|_{D} \rightarrow 0(h \rightarrow 0)$.

Proof. From $V \stackrel{c}{\hookrightarrow} D$, we derive that $T: D \rightarrow D$ is a completely continuous operator. It is obvious that $T_{h}: D \rightarrow D$ is a finite rank operator. From (2.12), (2.26), and (2.27), we deduce

$$
\left\|T f-T_{h} f\right\|_{a} \leq C_{e}\left(\inf _{q \in V_{h}}\|T f-q\|_{a}+\inf _{v \in W_{h}}\|S f-v\|_{W}\right) \longrightarrow 0 \quad(h \longrightarrow 0) .
$$

It shows that $T_{h}: D \rightarrow V$ pointwisely converges to $T$. From (2.10) and (2.12) we derive that both $T: D \rightarrow V$ and $T_{h}: D \rightarrow V$ are linear bounded. Hence, from Banach-Steinhaus Theorem, we know that there exists a positive constant $M$ independent of $h$, such that

$$
\sup _{h}\left\|T_{h}\right\|_{L(D, V)} \leq M
$$

Thus, $\cup_{h>0}\left(T-T_{h}\right) B$ is a bounded set in $V$ with respect to the unit ball $B$ of $D$. From $V \stackrel{c}{\hookrightarrow} D$, we know that $\cup_{h>0}\left(T-T_{h}\right) B$ is a relatively compact set in $D$, which proves that $\left\{T_{h}\right\}$ is collectively compact. From (2.28), we know that $T: D \rightarrow D, T_{h}: D \rightarrow D$, and $T_{h}$ pointwisely converge to $T$. From [7], $T: D \rightarrow D, T_{h}: D \rightarrow D$ are self-adjoint operators in the sense of inner product $(\cdot, \cdot)_{D}$. Then by Lemma 3.7 or Table 3.1 in [17], we get $\left\|T_{h}-T\right\|_{D} \rightarrow 0(h \rightarrow 0)$. The proof is completed. 
Lemma 2.3. Suppose that the conditions of Theorem 2.2 hold. Let $\left(\lambda_{h}, u_{h}, \sigma_{h}\right)$ be the kth eigenpair of (2.5)-(2.6) with $\left\|u_{h}\right\|_{a}=1$, let $\lambda$ be the kth eigenvalue of (2.3)-(2.4). Then $\lambda_{h} \rightarrow \lambda(h \rightarrow 0)$, and there exists an eigenfunction $(u, \sigma)$ corresponding to $\lambda$, such that

$$
\begin{gathered}
\left|\lambda_{h}-\lambda\right|+\left\|u_{h}-u\right\|_{D} \leq C_{1}\left\|\left.\left(T-T_{h}\right)\right|_{M(\lambda)}\right\|_{D^{\prime}} \\
\left\|\sigma-\sigma_{h}\right\|_{W} \leq\left\|S_{h}(\lambda u)-S(\lambda u)\right\|_{W}+C\left\|\left.\left(T-T_{h}\right)\right|_{M(\lambda)}\right\|_{D^{\prime}} \\
\left\|u-u_{h}\right\|_{a} \leq C_{2}\left\|\left.\left(T_{h}-T\right)\right|_{M(\lambda)}\right\|_{a^{\prime}}
\end{gathered}
$$

let $u \in \widehat{M}(\lambda)$, then there exists $u_{h} \in M_{h}(\lambda)$ such that

$$
\left\|u-u_{h}\right\|_{a} \leq C_{3}\left\|\left.\left(T_{h}-T\right)\right|_{M(\lambda)}\right\|_{a^{\prime}}
$$

where $u$ depends on $h$ in general, and $C_{1}, C_{2}$, and $C_{3}$ are constants independent of $h$.

Proof. By Theorem 2.2, we know $\left\|T_{h}-T\right\|_{D} \rightarrow 0(h \rightarrow 0)$. Thus from Theorem 2.2 in [7], we see that the desired results are valid. The proof is completed.

For $\left(u^{*}, \sigma^{*}\right) \in V \times W, u^{*} \neq 0$, define the Rayleigh quotient

$$
\lambda^{r}=\frac{a\left(u^{*}, u^{*}\right)+2 b\left(u^{*}, \sigma^{*}\right)}{\left(u^{*}, u^{*}\right)_{D}}
$$

Lemma 2.4. Suppose $(\lambda, u, \sigma)$ is an eigenpair of (2.3)-(2.4), then for any $\left(u^{*}, \sigma^{*}\right) \in V \times W, u^{*} \neq 0$, the Rayleigh quotient $\lambda^{r}$ satisfies

$$
\lambda^{r}-\lambda=\frac{a\left(u^{*}-u, u^{*}-u\right)+2 b\left(u^{*}-u, \sigma^{*}-\sigma\right)}{\left(u^{*}, u^{*}\right)_{D}}-\lambda \frac{\left(u^{*}-u, u^{*}-u\right)_{D}}{\left(u^{*}, u^{*}\right)_{D}} .
$$

Proof. The proof is completed by using the same argument as that of Lemma 9.1 (see [7, 18]). such that

Since $V \hookrightarrow D$ (continuously imbedded), there exists a constant $C_{4}$ independent of $h$

$$
\|v\|_{D} \leq C_{4}\|v\|_{a}, \quad \forall v \in V
$$

Taking $\left(u^{*}, \sigma^{*}\right)=\left(u_{h}, \sigma_{h}\right)$ in (2.35) and using (2.4) and (2.6), we deduce the following. 
Lemma 2.5. Suppose $\left(\lambda_{h}, u_{h}, \sigma_{h}\right)$ is an approximation of $(\lambda, u, \sigma)$ and $\left\|u_{h}\right\|_{a}=1$, then

$$
\begin{aligned}
\lambda_{h}-\lambda= & \frac{a\left(u_{h}-u, u_{h}-u\right)+2 b\left(u_{h}-u, v-\sigma\right)}{\left(u_{h}, u_{h}\right)_{D}} \\
& -\lambda \frac{\left(u_{h}-u, u_{h}-u\right)_{D}}{\left(u_{h}, u_{h}\right)_{D}}, \quad \forall v \in W_{h}, \\
\left|\lambda_{h}-\lambda\right| \leq & \left(\lambda_{h}+\lambda \lambda_{h} C_{4}^{2}\right)\left\|u_{h}-u\right\|_{a}^{2} \\
& +2 \lambda_{h} M_{2}\left\|u_{h}-u\right\|_{V}\|\sigma-v\|_{W}, \quad \forall v \in W_{h} .
\end{aligned}
$$

\section{Mixed Finite Element Multigrid Discretization Scheme Based on the Rayleigh Quotient Iteration}

In this section, we develop the work in [8], noticing that in [8], when $k>1, \lambda_{k}^{h_{1}}-\lambda_{k}, \lambda_{k}^{h_{l-1}}-$ $\lambda_{k, h_{l}}$ and $\lambda_{k}^{h_{1-1}}-\lambda_{k}$ sholud be modified to their absolute values, respectively, and establish the following mixed finite element multi-grid discretization scheme based on the Rayleigh quotient iteration, and give a rigorous theoretical analysis. Suppose the partition satisfies the following conditions.

Condition 1. $\left\{K^{h_{i}}\right\}$ is a family of regular meshes (see [19]) with the mesh diameter $\left\{h_{i}\right\}$ and $h_{i}=h_{i-1}^{t_{i}}, t_{i} \in(1,3)$ is arbitrarily chosen, $i=1,2, \ldots$, and $\inf _{i} t_{i}>1$.

Let $\left\{V_{h_{i}}\right\}_{0}^{l}$ and $\left\{W_{h_{i}}\right\}_{0}^{l}$ be finite element spaces on $\left\{K^{h_{i}}\right\}_{0}^{l}$.

Scheme 1. Multigrid Discretization.

Step 1. Solve the eigenvalue problem (2.3)-(2.4) on $V_{H} \times W_{H}$ : find $\left(\lambda_{H}, u_{H}, \sigma_{H}\right) \in R \times V_{H} \times W_{H}$, $\left\|u_{H}\right\|_{a}=1$ such that

$$
\begin{gathered}
a\left(u_{H}, \psi\right)+b\left(\psi, \sigma_{H}\right)=\lambda_{H}\left(u_{H}, \psi\right)_{D^{\prime}} \quad \forall \psi \in V_{H}, \\
b\left(u_{H}, v\right)=0, \quad \forall v \in W_{H} .
\end{gathered}
$$

Step 2. $u^{h_{0}} \Leftarrow u_{H}, \lambda^{h_{0}} \Leftarrow \lambda_{H}, i \Leftarrow 1$.

Step 3. Solve an equation on $V_{h_{i}} \times W_{h_{i}}$ : find $\left(u^{\prime}, \sigma^{\prime}\right) \in V_{h_{i}} \times W_{h_{i}}$ such that

$$
\begin{gathered}
a\left(u^{\prime}, \psi\right)+b\left(\psi, \sigma^{\prime}\right)-\lambda^{h_{i-1}}\left(u^{\prime}, \psi\right)_{D}=\left(u^{h_{i-1}}, \psi\right)_{D^{\prime}} \quad \forall \psi \in V_{h_{i}}, \\
b\left(u^{\prime}, v\right)=0, \quad \forall v \in W_{h_{i}} .
\end{gathered}
$$

Set $u^{h_{i}}=u^{\prime} /\left\|u^{\prime}\right\|_{a^{\prime}} \sigma^{h_{i}}=\sigma^{\prime} /\left\|u^{\prime}\right\|_{a}$. 
Step 4. Compute the Rayleigh quotient

$$
\lambda^{h_{i}}=\frac{a\left(u^{h_{i}}, u^{h_{i}}\right)}{\left(u^{h_{i}}, u^{h_{i}}\right)_{D}}
$$

Step 5. If $i=l$, then output $\left(\lambda^{h_{l}}, u^{h_{l}}, \sigma^{h_{l}}\right)$, stop; else, $i \Leftarrow i+1$, and return to Step 3.

Let $\left(\lambda_{H}, u_{H}, \sigma_{H}\right)$ be the $k$ th eigenpair, and we use $\left(\lambda^{h_{l}}, u^{h_{l}}, \sigma^{h_{l}}\right)$ as the $k$ th approximation eigenpair of (2.3)-(2.4).

Next, we will discuss the validity of Scheme 1.

Lemma 3.1. For any nonzero $u, v \in V$,

$$
\left\|\frac{u}{\|u\|_{a}}-\frac{v}{\|v\|_{a}}\right\|_{a} \leq 2 \frac{\|u-v\|_{a}}{\|u\|_{a}}, \quad\left\|\frac{u}{\|u\|_{a}}-\frac{v}{\|v\|_{a}}\right\|_{a} \leq 2 \frac{\|u-v\|_{a}}{\|v\|_{a}}
$$

Proof. See [8].

Denote $\operatorname{dist}(u, V)=\inf _{v \in V}\|u-v\|_{a}$.

Consider the eigenvalue problem (2.16) on $V_{h}$.

Lemma 3.2. Suppose that $\mu$ and $\mu_{h}$ are the $k$ th eigenvalue of $T$ and $T_{h}$, respectively, and $\left(\mu_{0}, u_{0}\right)$ is an approximate eigenpair, where $\mu_{0}$ is not an eigenvalue of $T_{h}, u_{0} \in V_{h},\left\|u_{0}\right\|_{a}=1, \operatorname{dist}\left(u_{0}, M_{h}(\mu)\right) \leq$ $1 / 2, \max _{k \leq j \leq k+q-1}\left|\left(\mu_{j, h}-\mu_{h}\right) /\left(\mu_{0}-\mu_{j, h}\right)\right| \leq 1 / 2,\left|\mu_{0}-\mu_{j, h}\right| \geq(\rho / 2)(j \neq k, k+1, \ldots, k+q-1)$, and $u^{s} \in V_{h}, u^{h} \in V_{h}$ satisfy

$$
\left(\mu_{0}-T_{h}\right) u^{s}=u_{0}, \quad u^{h}=\frac{u^{s}}{\left\|u^{s}\right\|_{a}}
$$

Then

$$
\operatorname{dist}\left(u^{h}, \widehat{M}_{h}(\mu)\right) \leq \frac{16}{\rho}\left|\mu_{0}-\mu_{h}\right| \operatorname{dist}\left(u_{0}, M_{h}(\mu)\right),
$$

where $\rho=\min _{\mu_{j} \neq \mu}\left|\mu_{j}-\mu\right|$ is the separation constant of the eigenvalue $\mu$.

Proof. See [8]

Since the convergence rate of $V_{h_{l-1}}$ and $W_{h_{l-1}}$ approximating eigenfunctions is lower than that of $V_{h_{l}}$ and $W_{h_{l}}$ approximating eigenfunctions, respectively, the approximation order of $\left(\lambda_{h_{l-1}}, u_{h_{l-1}}\right)$ is lower than that of $\left(\lambda_{h_{l}}, u_{h_{l}}\right)$. However, in general, the accuracy order of $\left(\lambda^{h_{l-1}}, u^{h_{l-1}}\right)$ will not exceed that of $\left(\lambda_{h_{l-1}}, u_{h_{l-1}}\right)$; therefore in the following Theorem 3.3 we assume that the accuracy order of $\left(\lambda^{h_{l-1}}, u^{h_{l-1}}\right)$ is lower than that of $\left(\lambda_{h_{l}}, u_{h_{l}}\right)$.

Theorem 3.3. Suppose that $\left\|T_{h}-T\right\|_{D} \rightarrow 0(h \rightarrow 0), H$ is small properly, and Condition 1 holds. Let $\left(\lambda^{h_{l}}, u^{h_{l}}, \sigma^{h_{l}}\right)$ be the approximate eigenpair obtained by Scheme 1 , and let $u^{h_{l-1}}$ approximate 
$\bar{u} \in \widehat{M}(\lambda), \lambda^{h_{l-1}}$ approximate $\lambda$, and the accuracy order of $\left(\lambda^{h_{l-1}}, u^{h_{l-1}}\right)$ be lower than that of $\left(\lambda_{h_{l}}, u_{h_{l}}\right)$. Then there exists $u \in M(\lambda)$ such that

$$
\begin{aligned}
\left\|u^{h_{l}}-u\right\|_{a} \leq & \frac{32}{\rho} C_{5} C_{6}\left(\left|\lambda^{h_{l-1}}-\lambda\right|^{2}+\left|\lambda^{h_{l-1}}-\lambda\right|\left\|u^{h_{l-1}}-\bar{u}\right\|_{D}\right) \\
& +\left(C_{2} \times q+3\right)\left\|\left.\left(T-T_{h_{l}}\right)\right|_{M(\lambda)}\right\|_{a^{\prime}} \\
\left|\lambda^{h_{l}}-\lambda\right| \leq & \left(\lambda^{h_{l}}+\lambda \lambda^{h_{l}} C_{4}^{2}\right)\left\|u^{h_{l}}-u\right\|_{a}^{2}+2 \lambda^{h_{l}} M_{2}\left\|u^{h_{l}}-u\right\|_{V v \in W_{h_{l}}}\|\sigma-v\|_{W},
\end{aligned}
$$

where $C_{5}$ and $C_{6}$ are determined, respectively, by (3.10) and (3.14) in the following proof.

Proof. Let $\mu_{0}=1 / \lambda^{h_{l-1}}$, and $u_{0}=\lambda^{h_{l-1}} T_{h_{l}} u^{h_{l-1}} /\left\|\lambda^{h_{l-1}} T_{h_{l}} u^{h_{l-1}}\right\|_{a}$. Since $\bar{u} \in M(\lambda)$, by calculation we deduce

$$
\begin{aligned}
\left\|\lambda^{h_{l-1}} T_{h_{l}} u^{h_{l-1}}-\bar{u}\right\|_{a} & =\left\|\lambda^{h_{l-1}} T_{h_{l}} u^{h_{l-1}}-\lambda T \bar{u}\right\|_{a} \\
& =\left|\lambda^{h_{l-1}}-\lambda\right|\left\|T_{h_{l}} u^{h_{l-1}}\right\|_{a}+\lambda\left\|T_{h_{l}}\left(u^{h_{l-1}}-\bar{u}\right)\right\|_{a}+\lambda\left\|\left(T_{h_{l}}-T\right) \bar{u}\right\|_{a} .
\end{aligned}
$$

From Lemma 2.1, there exists a positive constant $C_{5}$ depending only on $v, v_{1}, v_{2}, M_{1}$, and $M_{2}$ such that

$$
\left\|T_{h_{l}} v\right\|_{a} \leq C_{5}\|v\|_{D}, \quad \forall v \in D
$$

Then

$$
\left\|\lambda^{h_{l-1}} T_{h_{l}} u^{h_{l-1}}-\bar{u}\right\|_{a} \leq C_{5}\left(C_{4}\left|\lambda^{h_{l-1}}-\lambda\right|+\lambda\left\|u^{h_{l-1}}-\bar{u}\right\|_{D}\right)+\lambda\left\|\left.\left(T_{h_{l}}-T\right)\right|_{M(\lambda)}\right\|_{a} .
$$

By Lemma 3.1, we derive

$$
\begin{aligned}
\operatorname{dist}\left(u_{0}, \widehat{M}(\lambda)\right) & \leq\left\|u_{0}-\bar{u}\right\|_{a} \leq 2\left\|\lambda^{h_{l-1}} T_{h_{l}} u^{h_{l-1}}-\bar{u}\right\|_{a} \\
& \leq 2 C_{5}\left(C_{4}\left|\lambda^{h_{l-1}}-\lambda\right|+\lambda\left\|u^{h_{l-1}}-\bar{u}\right\|_{D}\right)+2 \lambda\left\|\left.\left(T_{h_{l}}-T\right)\right|_{M(\lambda)}\right\|_{a} .
\end{aligned}
$$

Using the triangle inequality and (2.33), we deduce

$$
\operatorname{dist}\left(u_{0}, M_{h_{l}}(\lambda)\right) \leq \operatorname{dist}\left(u_{0}, \widehat{M}(\lambda)\right)+C_{3}\left\|\left.\left(T_{h_{l}}-T\right)\right|_{M(\lambda)}\right\|_{a}
$$

According to the hypotheses of the theorem, we know that $\lambda^{h_{l-1}} \rightarrow \lambda$ and $\lambda^{h_{l-1}}-\lambda$ are an infinitesimal of lower order comparing with $\lambda_{j, h_{l}}-\lambda$. Hence, there exists a positive constant $C_{6}$ independent of $h_{l}(l=1,2, \ldots)$ such that for $j=k, k+1, \ldots, k+q-1$ we have

$$
\left|\mu_{0}-\mu_{j, h_{l}}\right|=\left|\frac{\lambda^{h_{l-1}}-\lambda+\lambda-\lambda_{j, h_{l}}}{\lambda_{j, h_{l}} \lambda^{h_{l-1}}}\right| \leq C_{6}\left|\lambda^{h_{l-1}}-\lambda\right|
$$


Note that $H$ is small enough and $h_{l} \ll h_{l-1}$; from (3.13) and (3.12), we obtain

$$
\operatorname{dist}\left(u_{0}, M_{h_{l}}(\lambda)\right) \leq \frac{1}{2}
$$

Noticing that $\lambda=\lambda_{k}=\lambda_{k+1}=\cdots=\lambda_{k+q-1}$, we have

$$
\left|\mu_{j, h_{l}}-\mu_{h_{l}}\right|=\left|\frac{\lambda_{h_{l}}-\lambda_{j, h_{l}}}{\lambda_{h_{l}} \lambda_{j, h_{l}}}\right|=\left|\frac{\lambda_{h_{l}}-\lambda+\lambda_{j}-\lambda_{j, h_{l}}}{\lambda_{h_{l}} \lambda_{j, h_{l}}}\right|,
$$

which together with (3.14), noting that $\lambda_{j, h_{l}}-\lambda$ is an infinitesimal of higher order comparing with $\lambda^{h_{l-1}}-\lambda$, yields

$$
\max _{k \leq j \leq k+q-1}\left|\frac{\mu_{j, h_{l}}-\mu_{h_{l}}}{\mu_{0}-\mu_{j, h_{l}}}\right| \leq \frac{1}{2} .
$$

Since $\rho$ is the separation constant, $H$ is small enough, and $h_{l} \ll h_{l-1}$, there holds

$$
\left|\mu_{0}-\mu_{j, h_{l}}\right| \geq \frac{\rho}{2}, \quad j \neq k, k+1, \ldots, k+q-1
$$

For $u^{\prime}$ in Step 3 of Scheme 1, from the definition of $T_{h}$ and $S_{h}$ (taking $i=l$ ), we have

$$
\begin{gathered}
a\left(\lambda^{h_{l-1}} T_{h_{l}} u^{\prime}, \psi\right)+b\left(\psi, \lambda^{h_{l-1}} S_{h_{l}} u^{\prime}\right)=\lambda^{h_{l-1}}\left(u^{\prime}, \psi\right)_{D^{\prime}} \quad \forall \psi \in V_{h_{l}} \\
b\left(\lambda^{h_{l-1}} T_{h_{l}} u^{\prime}, v\right)=0, \quad \forall v \in W_{h_{l}}, \\
a\left(T_{h_{l}} u^{h_{l-1}}, \psi\right)+b\left(\psi, S_{h_{l}} u^{h_{l-1}}\right)=\left(u^{h_{l-1}}, \psi\right)_{D^{\prime}} \quad \forall \psi \in V_{h_{l}} \\
b\left(T_{h_{l}} u^{h_{l-1}}, v\right)=0, \quad \forall v \in W_{h_{l}} .
\end{gathered}
$$

Hence, when $i=l$, Step 3 of Scheme 1 is equivalent to the following: find $\left(u^{\prime}, \sigma^{\prime}\right) \in V_{h_{l}} \times W_{h_{l}}$ such that

$$
\begin{gathered}
a\left(u^{\prime}, \psi\right)+b\left(\psi, \sigma^{\prime}\right)-\lambda^{h_{l-1}} a\left(T_{h_{l}} u^{\prime}, \psi\right)-\lambda^{h_{l-1}} b\left(\psi, S_{h_{l}} u^{\prime}\right) \\
=a\left(T_{h_{l}} u^{h_{l-1}}, \psi\right)+b\left(\psi, S_{h_{l}} u^{h_{l-1}}\right), \quad \forall \psi \in V_{h_{l}}, \\
b\left(u^{\prime}, v\right)=0, \quad \forall v \in W_{h_{l} .}
\end{gathered}
$$

And set $u^{h_{l}}=u^{\prime} /\left\|u^{\prime}\right\|_{a^{\prime}} \sigma^{h_{l}}=\sigma^{\prime} /\left\|u^{\prime}\right\|_{a}$.

From (3.23), we obtain

$$
a\left(u^{\prime}-\lambda^{h_{l-1}} T_{h_{l}} u^{\prime}-T_{h_{l}} u^{h_{l-1}}, \psi\right)+b\left(\psi, \sigma^{\prime}-\lambda^{h_{l-1}} S_{h_{l}} u^{\prime}-S_{h_{l}} u^{h_{l-1}}\right)=0, \quad \forall \psi \in V_{h_{l}} .
$$


Combining (3.24), (3.20) and (3.22), we get

$$
b\left(u^{\prime}-\lambda^{h_{l-1}} T_{h_{l}} u^{\prime}-T_{h_{l}} u^{h_{l-1}}, v\right)=0, \quad \forall v \in W_{h_{l}}
$$

By (3.26) and taking $\psi=u^{\prime}-\lambda^{h_{l-1}} T_{h_{l}} u^{\prime}-T_{h_{l}} u^{h_{l-1}}$ in (3.25), we obtain

$$
a\left(u^{\prime}-\lambda^{h_{l-1}} T_{h_{l}} u^{\prime}-T_{h_{l}} u^{h_{l-1}}, u^{\prime}-\lambda^{h_{l-1}} T_{h_{l}} u^{\prime}-T_{h_{l}} u^{h_{l-1}}\right)=0
$$

Thus

$$
\left(\frac{1}{\lambda^{h_{l-1}}}-T_{h_{l}}\right) u^{\prime}=\frac{1}{\lambda^{h_{l-1}}} T_{h_{l}} u^{h_{l-1}}, \quad u^{h_{l}}=\frac{u^{\prime}}{\left\|u^{\prime}\right\|_{a}} .
$$

From (3.28) we know that the first term on the left-hand side of (3.25) is equal to 0, thus

$$
b\left(\psi, \sigma^{\prime}-\lambda^{h_{l-1}} S_{h_{l}} u^{\prime}-S_{h_{l}} u^{h_{l-1}}\right)=0, \quad \forall \psi \in V_{h_{l}}
$$

then, using discrete inf-sup condition we get

$$
\sigma^{\prime}=\lambda^{h_{l-1}} S_{h_{l}} u^{\prime}+S_{h_{l}} u^{h_{l-1}} .
$$

Thus Step 3 of Scheme 1 is equivalent to (3.28), (3.30), $u^{h_{l}}=u^{\prime} /\left\|u^{\prime}\right\|_{a^{\prime}}$ and $\sigma^{h_{l}}=\sigma^{\prime} /\left\|u^{\prime}\right\|_{a}$.

Noting that $\left(1 / \lambda^{h_{l-1}}\right) T_{h_{l}} u^{h_{l-1}}=\left\|\left(1 / \lambda^{h_{l-1}}\right) T_{h_{l}} u^{h_{l-1}}\right\|_{a} u_{0}$ differs from $u_{0}$ by only a constant and selecting $u^{s}=\left(\lambda^{h_{l-1}} u^{\prime}\right) /\left\|T_{h_{l}} u^{h_{l-1}}\right\|_{a^{\prime}}$, we have

$$
\left(\frac{1}{\lambda^{h_{l-1}}}-T_{h_{l}}\right) u^{S}=u_{0}, \quad u^{h_{l}}=\frac{u^{S}}{\left\|u^{s}\right\|_{a}}
$$

By (3.15), (3.17), (3.18), and (3.31), we see that the conditions of Lemma 3.2 hold. Thus, substituting (3.13) and (3.14) into (3.6), we obtain

$$
\operatorname{dist}\left(u^{h_{l}}, \widehat{M}_{h_{l}}(\lambda)\right) \leq \frac{16}{\rho} C_{6}\left(\lambda^{h_{l-1}}-\lambda\right)\left(\operatorname{dist}\left(u_{0}, \widehat{M}(\lambda)\right)+C_{3}\left\|\left.\left(T-T_{h_{l}}\right)\right|_{M(\lambda)}\right\|_{a}\right)
$$

Let the eigenfunctions $\left\{u_{j, h_{l}}\right\}_{k}^{k+q-1}$ be an orthonormal system of $M_{h_{l}}(\lambda)$ in the sense of $a(\cdot, \cdot)$, then

$$
\operatorname{dist}\left(u^{h_{l}}, M_{h_{l}}(\lambda)\right)=\left\|u^{h_{l}}-\sum_{j=k}^{k+q-1} a\left(u^{h_{l}}, u_{j, h_{l}}\right) u_{j, h_{l}}\right\|_{a} .
$$


Let

$$
u^{*}=\sum_{j=k}^{k+q-1} a\left(u^{h_{l}}, u_{j, h_{l}}\right) u_{j, h_{l}}
$$

noting $\left\|u^{h_{l}}-u^{*}\right\|_{a} \leq \operatorname{dist}\left(u^{h_{l}}, \widehat{M}_{h_{l}}(\lambda)\right)$, from (3.32) we deduce

$$
\left\|u^{h_{l}}-u^{*}\right\|_{a} \leq \frac{16}{\rho} C_{6}\left(\lambda^{h_{l-1}}-\lambda\right)\left(\operatorname{dist}\left(u_{0}, \widehat{M}(\lambda)\right)+C_{3}\left\|\left.\left(T-T_{h_{l}}\right)\right|_{M(\lambda)}\right\|_{a}\right) .
$$

By Lemma 2.3, there exist $\left\{u_{j}^{0}\right\}_{k}^{k+q-1} \subset \widehat{M}(\lambda)$ such that $u_{j, h_{l}}-u_{j}^{0}$ satisfy (2.32). Let

$$
u=\sum_{j=k}^{k+q-1} a\left(u^{h_{l}}, u_{j, h_{l}}\right) u_{j}^{0}
$$

then $u \in M(\lambda)$. Using (2.32), we deduce

$$
\left\|u^{*}-u\right\|_{a}=\left\|\sum_{j=k}^{k+q-1} a\left(u^{h_{l}}, u_{j, h_{l}}\right)\left(u_{j, h_{l}}-u_{j}^{0}\right)\right\|_{a} \leq C_{2} q\left\|\left.\left(T_{h_{l}}-T\right)\right|_{M(\lambda)}\right\|_{a} .
$$

Combining (3.35) with the above inequality, we have

$$
\begin{aligned}
\left\|u^{h_{l}}-u\right\|_{a} \leq & \frac{16}{\rho} C_{6}\left(\lambda^{h_{l-1}}-\lambda\right) \operatorname{dist}\left(u_{0}, \widehat{M}(\lambda)\right) \\
& +\left(C_{2} q+1\right)\left\|\left.\left(T-T_{h_{l}}\right)\right|_{M(\lambda)}\right\|_{a} .
\end{aligned}
$$

Substituting (3.12) into (3.38), we get (3.7).

From Step 3 of Scheme 1, we know that $b\left(u^{h_{l}}, \sigma^{h_{l}}\right)=0$, thus

$$
\lambda^{h_{l}}=\frac{a\left(u^{h_{l}}, u^{h_{l}}\right)}{\left(u^{h_{l}}, u^{h_{l}}\right)_{D}}=\frac{a\left(u^{h_{l}}, u^{h_{l}}\right)+2 b\left(u^{h_{l}}, \sigma^{h_{l}}\right)}{\left(u^{h_{l}}, u^{h_{l}}\right)_{D}} .
$$

Select $\lambda^{r}=\lambda^{h_{l}}, u^{*}=u^{h_{l}}$, and $\sigma^{*}=\sigma^{h_{l}}$. From Lemma 2.4, we get

$$
\lambda^{h_{l}}-\lambda=\frac{a\left(u^{h_{l}}-u, u^{h_{l}}-u\right)+2 b\left(u^{h_{l}}-u, \sigma^{h_{l}}-\sigma\right)}{\left(u^{h_{l}}, u^{h_{l}}\right)_{D}}-\lambda \frac{\left(u^{h_{l}}-u, u^{h_{l}}-u\right)_{D}}{\left(u^{h_{l}}, u^{h_{l}}\right)_{D}} .
$$


Noting that $\forall v \in W_{h_{l}}, b\left(u^{h_{l}}-u, v\right)=0$, and $\left(u^{h_{l}}, u^{h_{l}}\right)_{D}=\left(1 / \lambda^{h_{l}}\right) a\left(u^{h_{l}}, u^{h_{l}}\right)=1 / \lambda^{h_{l}}$, we have

$$
\begin{aligned}
\lambda^{h_{l}}-\lambda= & \lambda^{h_{l}} a\left(u^{h_{l}}-u, u^{h_{l}}-u\right)+2 \lambda^{h_{l}} b\left(u^{h_{l}}-u, v-\sigma\right) \\
& -\lambda \lambda^{h_{l}}\left(u^{h_{l}}-u, u^{h_{l}}-u\right)_{D^{\prime}} \quad \forall v \in W_{h_{l}} .
\end{aligned}
$$

Note $\left\|u^{h_{l}}-u\right\|_{D} \leq C_{4}\left\|u^{h_{l}}-u\right\|_{a}$. From (3.41) we obtain (3.8).

For $l=1$, Scheme 1 is actually the two-grid discretization scheme established in [7]. By Theorem 3.3 we get the following conclusion directly.

Theorem 3.4. Suppose $\left\|T_{h}-T\right\|_{D} \rightarrow 0(h \rightarrow 0)$. Let $h_{0}=H$ be small properly. Let $\left(\lambda^{h_{1}}, u^{h_{1}}, \sigma^{h_{1}}\right)$ be an approximate eigenpair obtained by Scheme $1(l=1)$. Then there exists $u \in M(\lambda)$ such that

$$
\begin{aligned}
\left\|u^{h_{1}}-u\right\|_{a} \leq & \frac{32}{\rho} C_{5} C_{6}\left(\left|\lambda^{h_{0}}-\lambda\right|^{2}+\left|\lambda^{h_{0}}-\lambda\right| C_{1}\left\|\left.\left(T-T_{h_{0}}\right)\right|_{M(\lambda)}\right\|_{D}\right) \\
& +\left(C_{2} \times q+3\right)\left\|\left.\left(T-T_{h_{1}}\right)\right|_{M(\lambda)}\right\|_{a^{\prime}} \\
\left|\lambda^{h_{1}}-\lambda\right| \leq & \left(\lambda^{h_{1}}+\lambda \lambda^{h_{1}} C_{4}^{2}\right)\left\|u^{h_{1}}-u\right\|_{a}^{2}+2 \lambda^{h_{1}} M_{2}\left\|u^{h_{1}}-u\right\|_{V v \in W_{h_{1}}}\|\sigma-v\|_{W} .
\end{aligned}
$$

Proof. Consider Scheme 1. Here $l=1, u^{h_{0}}=u_{H}$. By Lemma 2.3, we know that $u^{h_{0}}$ approximates $\bar{u} \in \widehat{M}(\lambda)$, and the accuracy order of $\left(\lambda^{h_{0}}, u^{h_{0}}\right)$ is lower than $\left(\lambda_{h_{1}}, u_{h_{1}}\right)$. Hence, for $l=1$, the conditions of Theorem 3.3 hold. Select $\bar{u}$ in the proof of Theorem 3.3 such that $u^{h_{0}}-\bar{u}$ satisfies Lemma 2.3. Then from (2.30), we obtain

$$
\left\|u^{h_{0}}-\bar{u}\right\|_{D} \leq C_{1}\left\|\left.\left(T-T_{h_{0}}\right)\right|_{M(\lambda)}\right\|_{D^{\prime}}
$$

substituting it into (3.7), we obtain (3.42). From (3.8), we deduce (3.43).

Theorem 3.4 is actually Theorem 3.3 in [7], but we analyze in detail the constants appeared in the error estimates.

From Theorem 3.4 and Theorem 3.3, we know that $\lambda^{h_{l}} \rightarrow \lambda(l \rightarrow \infty)$ and the convergence rate is high. Thus, we use $\theta \times\left|\lambda^{h_{l}}-\lambda^{h_{l-1}}\right|$ as a posteriori error indicator of $\lambda^{h_{l}}-\lambda$ (details can be seen in Remark 4.5). Then we establish the following adaptive algorithm.

Scheme 2 (Adaptive Algorithm). Give an error tolerance $\varepsilon$ and choose the parameter $0<\theta \leq 1$, $H, t_{1}$, and $h_{1}=H^{t_{1}}$.

Step 1. Solve (2.3)-(2.4) on $V_{H} \times W_{H}$ : find $\left(\lambda_{H}, u_{H}, \sigma_{H}\right) \in R \times V_{H} \times W_{H},\left\|u_{H}\right\|_{a}=1$ such that

$$
\begin{gathered}
a\left(u_{H}, \psi\right)+b\left(\psi, \sigma_{H}\right)=\lambda_{H}\left(u_{H}, \psi\right)_{D^{\prime}} \quad \forall \psi \in V_{H}, \\
b\left(u_{H}, v\right)=0, \quad \forall v \in W_{H} .
\end{gathered}
$$

Step 2. $u^{h_{0}} \Leftarrow u_{H}, \lambda^{h_{0}} \Leftarrow \lambda_{H}, l \Leftarrow 1$. 
Step 3. Solve an equation on $V_{h_{l}} \times \mathrm{W}_{h_{l}}$ : find $\left(u^{\prime}, \sigma^{\prime}\right) \in V_{h_{l}} \times W_{h_{l}}$ such that

$$
\begin{gathered}
a\left(u^{\prime}, \psi\right)+b\left(\psi, \sigma^{\prime}\right)-\lambda^{h_{l-1}}\left(u^{\prime}, \psi\right)_{D}=\left(u^{h_{l-1}}, \psi\right)_{D^{\prime}} \quad \forall \psi \in V_{h_{l}} \\
b\left(u^{\prime}, v\right)=0, \quad \forall v \in W_{h_{l}} .
\end{gathered}
$$

Let $u^{h_{l}}=u^{\prime} /\left\|u^{\prime}\right\|_{a}, \sigma^{h_{l}}=\sigma^{\prime} /\left\|u^{\prime}\right\|_{a}$.

Step 4. Compute the Rayleigh quotient

$$
\lambda^{h_{l}}=\frac{a\left(u^{h_{l}}, u^{h_{l}}\right)}{\left(u^{h_{l}}, u^{h_{l}}\right)_{D}} .
$$

Step 5. If $\theta \times\left|\lambda^{h_{l}}-\lambda^{h_{l-1}}\right|>\varepsilon$, then select $t_{l+1}, h_{l+1}=h_{l}^{t_{l+1}}, l \Leftarrow l+1$ and return to Step 3; else output $\left(\lambda^{h_{l}}, u^{h_{l}}, \sigma^{h_{l}}\right)$, stop.

Remark 3.5. In Scheme 2, we use $\theta \times\left|\lambda^{h_{l}}-\lambda^{h_{l-1}}\right|$ as a posteriori error indicator which is global. In order to cope with difficulties caused by local singularity of a complicated problem in calculation, so far, most algorithms designed a local a posteriori error indicator to establish adaptive algorithm with local mesh refinement (e.g., see [20-22]). However, because the eigenvalue problem of electric field is so complicated, that it is very difficult to obtain a local a posteriori error indicator of eigenfunction. Fortunately, the influence of local singularity can be avoided by using the weight method which is established by Costabel and Dauge to discrete the eigenvalue problem of electric field. And the performance of the weigh method is very good (see $[3,11]$ ). Hence, without local mesh refinement, by using the weight method mentioned above our algorithm can also guarantee its high efficiency.

\section{The Eigenvalue Problem of Electric Field}

Consider the eigenvalue problem of electric field:

$$
\begin{gathered}
c^{2} \text { curlcurl } \vec{u}=\omega^{2} \vec{u}, \quad \text { in } \Omega, \\
\operatorname{div} \vec{u}=0, \quad \text { in } \Omega, \\
\vec{u} \times \vec{\gamma}=0, \quad \text { on } \in \partial \Omega,
\end{gathered}
$$

where $\Omega$ is a polyhedron in $\mathbf{R}^{3}, \vec{\gamma}$ is the unit outward normal to $\partial \Omega$.

Physically $\vec{u}$ denotes the electric field, $\omega$ denotes the time frequency, and $c$ is the speed of the light velocity. Usually, let $\lambda=\omega^{2} / c^{2}$ named eigenvalue.

Let

$$
\begin{aligned}
H(\operatorname{curl}, \Omega) & =\left\{\vec{q} \in L_{2}(\Omega)^{3}: \operatorname{curl} \vec{q} \in L_{2}(\Omega)^{3}\right\} \\
H_{0}(\operatorname{curl}, \Omega) & =\left\{\vec{q} \in H(\operatorname{curl}, \Omega): \vec{q} \times\left.\vec{\gamma}\right|_{\partial \Omega}=0\right\}
\end{aligned}
$$


When $\Omega$ is a convex polyhedron, we define the following function space:

$$
x=\left\{\vec{q} \in H_{0}(\text { curl }, \Omega): \operatorname{div} \vec{q} \in L^{2}(\Omega)\right\} .
$$

Denote

$$
\begin{gathered}
(\vec{q}, \vec{\psi})_{0}=\int_{\Omega} \vec{q} \cdot \vec{\psi} \mathrm{d} x, \quad\|\vec{q}\|_{0}=(\vec{q}, \vec{q})_{0}^{1 / 2} . \\
(\vec{q}, \vec{\psi})_{X}=(\operatorname{curl} \vec{q}, \operatorname{curl} \vec{\psi})_{0}+(\operatorname{div} \vec{q}, \operatorname{div} \vec{\psi})_{0}, \quad\|\vec{q}\|_{X}=(\vec{q}, \vec{q})_{X}^{1 / 2},
\end{gathered}
$$

From $[23,24]$, we know that $\chi \subset H^{1}(\Omega)^{3} ;(\vec{q}, \vec{\psi})_{X}$ is a coercive bilinear form on $\chi$, and $\|\vec{q}\|_{X}$ is a norm.

When $\Omega$ is a nonconvex polyhedron, the problem is relatively complicated. Let $E$ denote a set of edges of reentrant dihedral angles on $\partial \Omega$, and $d=d(x)$ denote the distance to the set $E: d(x)=\operatorname{dist}\left(x, \cup_{e \in E} \bar{e}\right)$. We introduce a weight function $\omega_{r}$ which is a nonnegative smooth function of $x$. It can be represented by $d^{r}$ in reentrant edge and angular domain. We shall write $\omega_{r} \simeq d^{r}$. Define the weighted functional spaces:

$$
\begin{aligned}
& L_{r}^{2}(\Omega)=\left\{v \in L_{\mathrm{loc}}^{2}(\Omega): \omega_{r} v \in L_{2}(\Omega)\right\} \\
& X_{r}=\left\{\vec{q} \in H_{0}(\operatorname{curl}, \Omega): \operatorname{div} \vec{q} \in L_{r}^{2}(\Omega)\right\} .
\end{aligned}
$$

Denote

$$
\begin{gathered}
(\vec{q}, \vec{\psi})_{L_{r}^{2}}=\int_{\Omega} \omega_{r}^{2} \vec{q} \cdot \vec{\psi} \mathrm{d} x, \quad\|\vec{q}\|_{L_{r}^{2}}=(\vec{q}, \vec{q})_{L_{r}^{2}}^{1 / 2} . \\
(\vec{q}, \vec{\psi})_{X_{r}}=(\operatorname{curl} \vec{q}, \operatorname{curl} \vec{\psi})_{0}+(\operatorname{div} \vec{q}, \operatorname{div} \vec{\psi})_{L_{r}^{2},} \quad\|\vec{q}\|_{X_{r}}=(\vec{q}, \vec{q})_{X_{r}}^{1 / 2} .
\end{gathered}
$$

Let $\sigma_{\Delta}^{D}$ be the following smallest singular exponent in the Laplace problem with homogenous Dirichlet boundary condition:

$$
\begin{gathered}
\left\{\phi \in H_{0}^{1}(\Omega): \Delta \phi \in L_{2}(\Omega)\right\} \subset \cap_{s<\sigma_{\Delta}^{D}} H^{s}(\Omega), \\
\left\{\phi \in H_{0}^{1}(\Omega): \Delta \phi \in L_{2}(\Omega)\right\} \ell H^{\sigma_{\Delta}^{D}}(\Omega) .
\end{gathered}
$$

From the regularity estimate, we know $\sigma_{\Delta}^{D} \in((3 / 2), 2)$. Let $r_{\min }=2-\sigma_{\Delta}^{D}$.

From $[11,25]$, we see that for all $r \in\left(r_{\min }, 1\right)$, the seminorm $\|\vec{q}\|_{\chi_{r}}$ is a norm on $\chi_{r}$, and $\chi_{r} \cap H^{1}(\Omega)^{3}$ is dense in $\chi_{r}$.

In the following discussion, we will use $\chi_{r}, L_{r}^{2}(\Omega)$ for both nonconvex and convex domains. We select $r \in\left(r_{\min }, 1\right)$ for non-convex domain and $\chi_{r}=X_{,} L_{r}^{2}(\Omega)=L^{2}(\Omega)$ for convex domain. 
By introducing the Lagrange multiplier $\sigma$, [6] changed (4.1) into the mixed variational formulation: find $(\lambda, \vec{u}, \sigma) \in \mathbf{R}^{+} \times X_{r} \times L_{r}^{2}(\Omega)$ such that

$$
\begin{gathered}
(\vec{u}, \vec{\psi})_{X_{r}}+(\operatorname{div} \vec{\psi}, \sigma)_{L_{r}^{2}}=\lambda(\vec{u}, \vec{\psi})_{0}, \quad \forall \vec{\psi} \in X_{r}, \\
(\operatorname{div} \vec{u}, v)_{L_{r}^{2}}=0, \quad \forall v \in L_{r}^{2}(\Omega) .
\end{gathered}
$$

Let $K^{h}$ be a regular simplex partition (tetrahedral partition) of $\Omega$ with the mesh diameter $h$. Define the finite element space $V_{h} \times W_{h} \subset X_{r} \times L_{r}^{2}(\Omega)$.

Restricting (4.8) on the above-mentioned finite space, we obtain the discrete mixed variational form: find $\left(\lambda_{h}, \vec{u}_{h}, \sigma_{h}\right) \in \mathbf{R}^{+} \times V_{h} \times W_{h}$ such that

$$
\begin{gathered}
\left(\vec{u}_{h}, \vec{\psi}\right)_{X_{r}}+\left(\operatorname{div} \vec{\psi}, \sigma_{h}\right)_{L_{r}^{2}}=\lambda_{h}\left(\vec{u}_{h}, \vec{\psi}\right)_{0^{\prime}} \quad \forall \vec{\psi} \in V_{h}, \\
\left(\operatorname{div} \vec{u}_{h}, v\right)_{L_{r}^{2}}=0, \quad \forall v \in W_{h} .
\end{gathered}
$$

Set

$$
\begin{gathered}
V=x_{r}, \quad\|\cdot\|_{V}=\|\cdot\|_{a}=\|\cdot\|_{X^{\prime}} \\
W=L_{r}^{2}(\Omega), \quad\|\cdot\|_{W}=\|\cdot\|_{L_{r}^{2}} \\
D=L_{2}(\Omega)^{3}, \quad\|\cdot\|_{D}=\|\cdot\|_{0} \\
a(\vec{q}, \vec{\psi})=(\vec{q}, \vec{\psi})_{X_{r}} b(\vec{\psi}, v)=(\operatorname{div} \vec{\psi}, v)_{L_{r}^{2}} .
\end{gathered}
$$

Then (4.8) and (4.9) can be written as (2.3)-(2.4) and (2.5)-(2.6), respectively (it is needed to add $\rightarrow$ for the vector function, e.g., $u, \psi$ should be written in the forms of $\vec{u}, \vec{\psi})$.

We apply Schemes 1 and 2 to the eigenvalue problem of electric field (4.8). Adding the symbol $\rightarrow$ for the vector function, we get a multi-grid discretization scheme and adaptive algorithm for mixed finite element of the eigenvalue problem of electric field which are still called Schemes 1 and 2.

It is easy to know that $a(\cdot, \cdot)$ and $b(\cdot, \cdot)$ are continuous bilinear forms on $V \times V$ and $V \times W$, respectively. $V \stackrel{c}{\hookrightarrow} D$. (It is true obviously when $\Omega$ is convex; when $\Omega$ is non-convex, see [25].)

Consider the source problem associated with (4.8): find $(\vec{w}, p) \in X_{r} \times L_{r}^{2}(\Omega)$ such that

$$
\begin{gathered}
(\vec{w}, \vec{\psi})_{X_{r}}+(\operatorname{div} \vec{\psi}, p)_{L_{r}^{2}}=(\vec{f}, \vec{\psi})_{0^{\prime}} \quad \forall \vec{\psi} \in X_{r}, \\
(\operatorname{div} \vec{w}, v)_{L_{r}^{2}}=0, \quad \forall v \in L_{r}^{2}(\Omega) .
\end{gathered}
$$

For (4.11) and its conforming finite element approximation, condition (C1) of Lemma 2.1 holds obviously; [3] has proved that condition (C2) holds; assume that the discrete inf-sup condition (C3) holds, then conditions of Lemma 2.1 hold. Thus we can define operators $T, S, T_{h}$, and $S_{h}$. (4.8) and (4.9) can be written as (2.15) and (2.16)-(2.17), respectively.

The following Lemma 4.1 is cited from $[3,25]$. 
Lemma 4.1. Equation (4.1) is equivalent to (4.8), and the solutions of (4.8) $\sigma=S(\lambda \vec{u})=0$ and $\vec{u} \in X_{r}, \operatorname{div} \vec{u}=0$.

Note that Lemma 4.1 shows that for the eigenvalue problem of electric field, the second term on the right-hand side in (3.8) is equal to 0 .

Theorem 4.2. Suppose that the discrete inf-sup condition (C3) holds. Then $\left\|T-T_{h}\right\|_{D} \rightarrow 0(h \rightarrow 0)$. Let $\left(\lambda_{h}, \vec{u}_{h}, \sigma_{h}\right)$ be the kth eigenpair of (4.9) with $\left\|\vec{u}_{h}\right\|_{X_{r}}=1$, and let $\lambda$ be the kth eigenvalue of (4.8). Then $\lambda_{h} \rightarrow \lambda(h \rightarrow 0)$, and there exists an eigenfunction $\vec{u}$ corresponding to $\lambda$ with $\|\vec{u}\|_{X_{r}}=1$, such that

$$
\begin{gathered}
\left|\lambda_{h}-\lambda\right| \leq\left(\lambda_{h}+\lambda \lambda_{h} C_{4}^{2}\right) C_{2}^{2}\left\|\left.\left(T_{h}-T\right)\right|_{M(\lambda)}\right\|_{X_{r^{\prime}}}^{2} \\
\left\|\vec{u}-\vec{u}_{h}\right\|_{\chi_{r}} \leq C_{2}\left\|\left.\left(T_{h}-T\right)\right|_{M(\lambda)}\right\|_{X_{r^{\prime}}}
\end{gathered}
$$

let $\vec{u} \in \widehat{M}(\lambda)$, then there exists $\vec{u}_{h} \in M_{h}(\lambda)$ such that

$$
\left\|\vec{u}-\vec{u}_{h}\right\|_{X_{r}} \leq C_{3}\left\|\left.\left(T_{h}-T\right)\right|_{M(\lambda)}\right\|_{X_{r}}
$$

where $C_{2}, C_{3}$, and $C_{4}$ are constants independent of $h$.

Proof. From the preceding discussion and hypotheses of the theorem, we know that $V \stackrel{c}{\hookrightarrow} D$, $a(u, v)$ is symmetric, and the conditions of Lemma 2.1 hold. Besides, since $K^{h}$ is a regular partition, when $\Omega$ is a convex polyhedron, $\chi_{r} \subset H^{1}(\Omega)^{3}\left(\chi_{r}=\chi\right)$; when $\Omega$ is a non-convex polyhedron, $x_{r} \cap H^{1}(\Omega)^{3}$ is dense in $x_{r}$. Since $C^{\infty}(\bar{\Omega})^{3}$ is dense in $H^{1}(\Omega)^{3}$, thus, no matter $\Omega$ is convex or non-convex, $C^{\infty}(\bar{\Omega})^{3}$ is dense in $\chi_{r}$. For any given $\vec{f} \in D$, we have $T \vec{f} \in X_{r}$. Thus for any $\varepsilon>0$, according to the density, we know that there exists $\vec{w} \in C^{\infty}(\bar{\Omega})^{3}$ such that

$$
\|T \vec{f}-\vec{w}\|_{\chi_{r}} \leq \frac{\varepsilon}{2}
$$

Selecting $h_{0}>0$ being small properly, when $0<h \leq h_{0}$, we have

$$
\left\|\vec{w}-I_{h} \vec{w}\right\|_{X_{r}} \leq C h|\vec{w}|_{2} \leq \frac{\varepsilon}{2}
$$

where $I_{h}: C^{\infty}(\bar{\Omega})^{3} \rightarrow V_{h}$ is an interpolation operator. Thus

$$
\begin{aligned}
\inf _{\vec{q} \in V_{h}}\|T \vec{f}-\vec{q}\|_{X_{r}} & \leq\left\|T \vec{f}-I_{h} \vec{w}\right\|_{X_{r}} \\
& \leq\|T \vec{f}-\vec{w}\|_{X_{r}}+\left\|\vec{w}-I_{h} \vec{w}\right\|_{X_{r}} \leq \frac{\varepsilon}{2}+\frac{\varepsilon}{2}=\varepsilon .
\end{aligned}
$$

Namely, $\inf _{\vec{q} \in V_{h}}\|T \vec{f}-\vec{q}\|_{X_{r}} \rightarrow 0(h \rightarrow 0)$. Hence (2.26) is true. Analogously, using the density of $C^{\infty}(\bar{\Omega})$ in $L_{r}^{2}(\Omega)$, we deduce that $\inf _{v \in W_{h}}\|S \vec{f}-v\|_{L_{r}^{2}} \rightarrow 0(h \rightarrow 0)$, namely, (2.27), is true. 
Hence, from Theorem 2.2 and Lemma 2.3, we know that $\left\|T-T_{h}\right\|_{D} \rightarrow 0(h \rightarrow 0), \lambda_{h} \rightarrow$ $\curlywedge(h \rightarrow 0),(4.13)$, and (4.14) hold. From (2.38), (4.13), and Lemma 4.1, we get (4.12). The proof is completed.

Denote

$$
\varepsilon_{\lambda}(h)=\sup _{\vec{u} \in \widehat{M}(\lambda)} \inf _{\vec{\psi} \in V_{h}}\|\vec{u}-\vec{\psi}\|_{X_{r}}
$$

From Lemma 2.1, noting $\sigma=0$, we deduce

$$
\begin{aligned}
\left\|\left.\left(T_{h}-T\right)\right|_{M(\lambda)}\right\|_{X_{r}} & =\sup _{\vec{u} \in \widehat{M}(\lambda)}\left\|\left(T_{h}-T\right) u\right\|_{X_{r}} \\
& \leq \sup _{\vec{u} \in \widehat{M}(\lambda)} C_{e} \inf _{\vec{\psi} \in V_{h}}\|T \vec{u}-\vec{\psi}\|_{X_{r}} \leq \lambda^{-1} C_{e} \varepsilon_{\lambda}(h) \equiv C_{7} \varepsilon_{\lambda}(h) .
\end{aligned}
$$

Theorem 4.3. Assume that the discrete inf-sup condition (C3) holds, $h_{0}=H$ is small properly, Condition 1 holds and $\sup _{i} t_{i}<3$. Let $\left(\lambda^{h_{l}}, u^{h_{l}}\right)$ be an approximate eigenpair obtained by Scheme 1, then there exists $\vec{u} \in M(\lambda)$ such that

$$
\begin{gathered}
\left\|\vec{u}^{h_{l}}-\vec{u}\right\|_{X_{r}} \leq 2\left(C_{2} \times q+3\right) C_{7} \varepsilon_{\lambda}\left(h_{l}\right), \\
\left|\lambda^{h_{l}}-\lambda\right| \leq 4\left(\lambda^{h_{l}}+\lambda \lambda^{h_{l}} C_{4}^{2}\right)\left(C_{2} \times q+3\right)^{2} C_{7}^{2} \varepsilon_{\lambda}\left(h_{l}\right)^{2} .
\end{gathered}
$$

Proof. We use induction to complete the proof. Note that the conditions of Theorem 2.2 hold. For $l=1$, Scheme 1 is actually two-grid discretization scheme. Substituting (4.19) into (4.12) and (4.13), we derive

$$
\begin{gathered}
\left\|\vec{u}_{H}-\vec{u}\right\|_{X_{r}} \leq C_{2} C_{7} \varepsilon_{\lambda}(H), \\
\left|\lambda-\lambda_{H}\right| \leq\left(\lambda_{H}+\lambda \lambda_{H} C_{4}^{2}\right) C_{2}^{2} C_{7}^{2} \varepsilon_{\lambda}(H)^{2} .
\end{gathered}
$$

Combining (3.7) with $l=1$ and the above two inequalities, we know that there exists $\vec{u} \in$ $M(\lambda)$ such that

$$
\begin{aligned}
\left\|u^{h_{1}}-\vec{u}\right\|_{X_{r}} \leq & \frac{32}{\rho} C_{5} C_{6}\left(\left|\lambda^{h_{0}}-\lambda\right|^{2}+\left|\lambda^{h_{0}}-\lambda\right|\left\|u^{h_{0}}-\bar{u}\right\|_{D}\right) \\
& +\left(C_{2} \times q+3\right)\left\|\left.\left(T-T_{h_{1}}\right)\right|_{M(\lambda)}\right\|_{X_{r}}
\end{aligned}
$$




$$
\begin{aligned}
& \leq \frac{32}{\rho} C_{5} C_{6}\left(\left(\lambda_{H}+\lambda \lambda_{H} C_{4}^{2}\right)^{2} C_{2}^{4} C_{7}^{4} \varepsilon_{\lambda}(H)^{4}\right. \\
& \left.\quad+\left(\lambda_{H}+\lambda \lambda_{H} C_{4}^{2}\right) C_{2}^{2} C_{7}^{2} \varepsilon_{\lambda}(H)^{2} C_{2} C_{4} C_{7} \varepsilon_{\lambda}(H)\right) \\
& \quad+\left(C_{2} \times q+3\right) C_{7} \varepsilon_{\lambda}\left(h_{1}\right) \\
& \leq 2\left(C_{2} \times q+3\right) C_{7} \varepsilon_{\lambda}\left(h_{1}\right) .
\end{aligned}
$$

Since $\sigma=0$, substituting (4.23) into (3.43), we deduce

$$
\begin{aligned}
\left|\lambda^{h_{1}}-\lambda\right| & \leq\left(\lambda^{h_{1}}+\lambda \lambda^{h_{1}} C_{4}^{2}\right)\left\|\vec{u}^{h_{1}}-\vec{u}\right\|_{x_{r}}^{2} \\
& \leq\left(\lambda^{h_{1}}+\lambda \lambda^{h_{1}} C_{4}^{2}\right) 4\left(C_{2} \times q+3\right)^{2} C_{7}^{2} \varepsilon_{\lambda}\left(h_{1}\right)^{2} .
\end{aligned}
$$

The above two inequalities show that Theorem 4.2 is true for $l=1$.

Suppose that the theorem is true for $l-1$, then by Theorem 3.3, we get

$$
\begin{aligned}
\left\|\vec{u}^{h_{l}}-\vec{u}\right\|_{X_{r}} \leq & \frac{32}{\rho} C_{5} C_{6}\left(\left(\lambda^{h_{l-1}}+\lambda \lambda^{h_{l-1}} C_{4}^{2}\right)^{2} 4^{2}\left(C_{2} \times q+3\right)^{4} C_{2}^{4} C_{7}^{4} \varepsilon_{\lambda}\left(h_{l-1}\right)^{4}\right. \\
& \left.\quad+\left(\lambda^{h_{l-1}}+\lambda \lambda^{h_{l-1}} C_{4}^{2}\right) 4\left(C_{2} \times q+3\right)^{2} C_{7}^{2} \varepsilon_{\lambda}\left(h_{l-1}\right)^{2} 2\left(C_{2} \times q+3\right) C_{7} \varepsilon_{\lambda}\left(h_{l-1}\right)\right) \\
& +\left(C_{2} \times q+3\right) C_{7} \varepsilon_{\lambda}\left(h_{l}\right) \\
\leq & 2\left(C_{2} \times q+3\right) C_{7} \varepsilon_{\lambda}\left(h_{l}\right),
\end{aligned}
$$

That is, (4.20) is valid.

From (4.20) and (3.8), we obtain (4.21). The proof is completed.

Assume that $K^{h}$ is a regular simplex partition (tetrahedral partition) of $\Omega$ with the mesh diameter $h$. Let $V_{h}$ and $W_{h}$ be the $P_{k+1}-P_{k}$ finite element spaces as follows:

$$
\begin{gathered}
V_{h}=\left\{\vec{q} \in C^{0}(\bar{\Omega})^{3}: \vec{q} \times\left.\vec{\gamma}\right|_{\partial \Omega}=0,\left.\vec{q}\right|_{\kappa} \in P_{k+1}(\kappa)^{3} \cdot \forall \kappa \in K^{h}\right\}, \\
W_{h}=\left\{v \in C^{0}(\bar{\Omega}):\left.v\right|_{\kappa} \in P_{k}(\mathcal{\kappa}), \forall \kappa \in \mathrm{K}^{h},\left.v\right|_{E_{h}}=0\right\} .
\end{gathered}
$$

Here we set $E_{h}=\cup_{\kappa \in K^{h}, \partial \kappa \cap E \neq \phi} \kappa .\left.v\right|_{E_{h}}=0$ means that $v$ is equal to 0 on the tetrahedron where reentrant edge and angular point are adjacent. Considering finite element approximation of (4.11), for the 3-D $P_{2}$-iso- $P_{1}$ Taylor-Hood finite element, Ciarlet and Girault [26] have discussed that the discrete inf-sup condition (C3) holds when $\Omega$ is a convex domain; for the $P_{k+1}-P_{k}$ element, Ciarlet and Hechme have proved that the discrete inf-sup condition (C3) holds when $\Omega$ is a polyhedron (see Section 2.2 in [4] and pp. 509 in [3]). 
From the above mentioned, we know that the $P_{k+1}-P_{k}$ element approximation of (4.11) satisfies the conditions of Theorem 4.2.

Let $\sigma_{\Delta}^{N}$ be the smallest singular exponent in the Laplace problem with homogenous Neumman boundary condition, then $\sigma_{\Delta}^{N} \in(3 / 2,2)$. Denote $\tau=\min \left(r-r_{\min }, \sigma_{\Delta}^{N}-1\right)$.

Corollary 4.4. Assume that $h_{0}=H$ is small properly, $t_{i} \in(1,3](i=1,2, ., l)$, and $h_{i}=h_{i-1}^{t_{i}}$ $(i=1,2, \ldots, l)$. Let $\left(\lambda^{h_{l}}, u^{h_{l}}\right)$ be an approximate eigenpair of the $P_{k+1}-P_{k}$ element obtained by Scheme 1 . Then when $\Omega$ is a convex domain, there exists $\vec{u} \in M(\lambda)$ such that

$$
\begin{gathered}
\left\|\vec{u}^{h_{l}}-\vec{u}\right\|_{X_{r}} \leq 2\left(C_{2} \times q+3\right) C_{7} C^{\prime} h_{l} \\
\left|\lambda^{h_{l}}-\lambda\right| \leq 4\left(\lambda^{h_{l}}+\lambda \lambda^{h_{l}} C_{4}^{2}\right)\left(C_{2} \times q+3\right)^{2} C_{7}^{2} C^{\prime 2} h_{l}^{2} .
\end{gathered}
$$

When $\Omega$ is a non-convex domain, there exists $\vec{u} \in M(\lambda)$ such that

$$
\begin{gathered}
\left\|\vec{u}^{h_{l}}-\vec{u}\right\|_{X_{r}} \leq 2\left(C_{2} \times q+3\right) C_{7} C^{\prime \prime} h_{l}^{\mu}, \quad \forall \mu \in(0, \tau), \\
\left|\lambda^{h_{l}}-\lambda\right| \leq 4\left(\lambda^{h_{l}}+\lambda \lambda^{h_{l}} C_{4}^{2}\right)\left(C_{2} \times q+3\right)^{2} C_{7}^{2} C^{\prime \prime 2} h_{l}^{2 \mu}, \quad \forall \mu \in(0, \tau),
\end{gathered}
$$

where $C^{\prime}$ and $C^{\prime \prime}$ are determined by (4.31) and (4.32) in the proof, respectively.

Proof. The hypotheses of Corollary 4.4 imply that the conditions of Theorem 4.3 hold. When $\Omega$ is convex, for any $\vec{u} \in M(\lambda)$ we have $\vec{u}=T(\lambda \vec{u}) \in H^{2}(\Omega)$ (see (44) in [1]). Thus there exists $C^{\prime}$ independent of $h_{l}(l=1,2, \ldots)$ such that

$$
\varepsilon_{\lambda}\left(h_{l}\right) \leq C^{\prime} h_{l}
$$

Substituting the above inequality into (4.20) and (4.21), we get (4.27) and (4.28), respectively. When $\Omega$ is a non-convex domain, for any $\vec{u} \in M(\lambda)$, by (36) in [3] we know that there exists $C^{\prime \prime}$ independent of $h_{l}(l=1,2, \ldots)$, such that

$$
\varepsilon_{\lambda}\left(h_{l}\right) \leq C^{\prime \prime} h_{l}^{\mu}
$$

Substituting the above inequality into (4.20) and (4.21), we derive (4.29) and (4.30), respectively.

Remark 4.5. From Corollary 4.4, we see that the constants in the error estimates are not only independent of the mesh diameter but also independent of the iterative degrees. Thus, when $l \rightarrow \infty$, we have $\lambda^{h_{l}} \rightarrow \lambda$. Suppose that the precision order of (4.28) is optimal which cannot be improved any more, then

$$
\begin{aligned}
& \lambda^{h_{l}}-\lambda^{h_{l+1}}=\lambda^{h_{l}}-\lambda+\lambda-\lambda^{h_{l+1}}=O\left(h_{l}^{2 r}\right), \\
& \lambda^{h_{l-1}}-\lambda^{h_{l}}=\lambda^{h_{l-1}}-\lambda+\lambda-\lambda^{h_{l}}=O\left(h_{l-1}^{2 r}\right),
\end{aligned}
$$




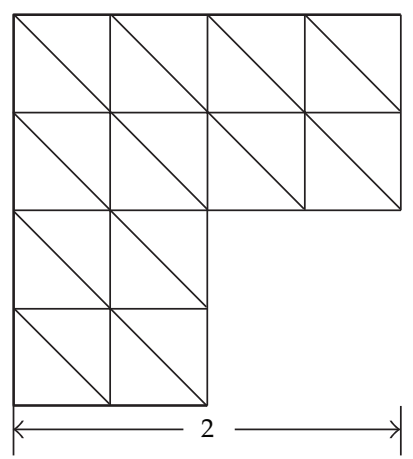

Figure 1

where for convex domain $r=1$, while for non-convex domain $r=\mu \in(0, \tau)$ but approximates $\tau$ arbitrarily. Therefore we have that $\lambda^{h_{l}}-\lambda^{h_{l+1}} \leq \lambda^{h_{l-1}}-\lambda^{h_{l}}$. Then

$$
\begin{aligned}
\left|\lambda^{h_{l}}-\lambda\right| & \leq\left|\lambda^{h_{l}}-\lambda^{h_{l+1}}\right|+\left|\lambda^{h_{l+1}}-\lambda\right| \\
& \leq\left|\lambda^{h_{l-1}}-\lambda^{h_{l}}\right|+\vartheta\left(h_{l+1}^{2 r}\right),
\end{aligned}
$$

thus we can use $\eta\left(\lambda^{h_{l}}\right)=\theta \times\left|\lambda^{h_{l-1}}-\lambda^{h_{l}}\right|$ as a posteriori error indicator of $\left|\lambda^{h_{l}}-\lambda\right|$, where $\theta \in(0,1]$.

\section{Numerical Experiments.}

Consider the eigenvalue problem (4.1) of electric field, where $\bar{\Omega}=[0, \pi] \times[0, \pi]$ is a square domain or $\bar{\Omega}=[-1,0] \times[-1,0] \cup[-1,1] \times[0,1]$ is an L-shaped domain. For the square domain, the first five exact eigenvalues are $\lambda_{1}=\lambda_{2}=1, \lambda_{3}=2$, and $\lambda_{4}=\lambda_{5}=4$; for the L-shaped domain, the first five eigenvalues are $\lambda_{1} \approx 1.47562182408, \lambda_{2} \approx 3.53403136678, \lambda_{3}=\lambda_{4}=\pi^{2} \approx$ 9.86960440109 , and $\lambda_{5} \approx 11.3894793979$, and the first eigenfunction has a strong singularity (see, e.g., [3]).

We adopt a uniform isosceles right triangulation for $\Omega$ (the edge in each element is along three fixed directions, see Figure 1) to produce the meshes $K^{h_{l}}$ with mesh diameter $h_{l}$.

The definition of $P_{2}-P_{1}$ mixed finite element spaces is given by

$$
\begin{gathered}
V_{h_{l}}=\left\{\vec{q} \in C^{0}(\bar{\Omega})^{2}: \vec{q} \times\left.\vec{\gamma}\right|_{\partial \Omega}=0,\left.\vec{q}\right|_{\kappa} \in P_{2}(\kappa)^{2}, \forall \kappa \in K^{h_{l}}\right\}, \\
W_{h_{l}}=\left\{v \in C^{0}(\bar{\Omega}):\left.v\right|_{\kappa} \in P_{1}(\kappa), \forall \kappa \in K^{h_{l}},\left.v\right|_{E_{h_{l}}}=0\right\} .
\end{gathered}
$$

We make use of Matlab to compute the first five approximate eigenvalues by using Scheme 1 with $P_{2}-P_{1}$ element. The numerical results are listed in Tables 1,2 , and 3. 
Table 1: The results on the square by Scheme $1\left(P_{2}-P_{1}\right.$ element) for the eigenvalue problem of electric field $(r=0)$.

\begin{tabular}{cccccccc}
\hline$k$ & $H$ & $l$ & $h_{l}$ & $\lambda_{k, h_{l}}$ & $\lambda_{k}^{h_{l}}$ & $\eta\left(\lambda_{k, h_{l}}\right) / e\left(\lambda_{k, h_{l}}\right)$ & $\eta\left(\lambda_{k}^{h_{l}}\right) / e\left(\lambda_{k}^{h_{l}}\right)$ \\
\hline 1 & $\sqrt{2} / 8$ & 1 & $\sqrt{2} / 32$ & 1.0000001280750 & 1.00000012807483 & 248.41 & 248.41 \\
1 & $\sqrt{2} / 8$ & 2 & $\sqrt{2} / 64$ & 1.0000000080355 & 1.00000000803501 & 14.94 & 14.94 \\
1 & $\sqrt{2} / 8$ & 3 & $\sqrt{2} / 128$ & - & 1.00000000050340 & - & 14.96 \\
3 & $\sqrt{2} / 8$ & 1 & $\sqrt{2} / 32$ & 2.0000017958515 & 2.00000179585163 & 245.62 & 245.62 \\
3 & $\sqrt{2} / 8$ & 2 & $\sqrt{2} / 64$ & 2.0000001126165 & 2.00000011261761 & 14.95 & 14.95 \\
3 & $\sqrt{2} / 8$ & 3 & $\sqrt{2} / 128$ & - & 2.00000000704840 & - & 14.98 \\
4 & $\sqrt{2} / 8$ & 1 & $\sqrt{2} / 32$ & 4.0000081820714 & 4.00000818207150 & 242.08 & 242.08 \\
4 & $\sqrt{2} / 8$ & 2 & $\sqrt{2} / 64$ & 4.0000005140035 & 4.00000051400385 & 14.92 & 14.92 \\
4 & $\sqrt{2} / 8$ & 3 & $\sqrt{2} / 128$ & - & 4.00000003219500 & - & 14.97 \\
\hline
\end{tabular}

Table 2: The results on the L-shaped domain by Scheme $1\left(P_{2}-P_{1}\right.$ element) for the eigenvalue problem of electric field $(r=0.5)$.

\begin{tabular}{cccccccc}
\hline$k$ & $H$ & $l$ & $h_{l}$ & $\lambda_{k, h_{l}}$ & $\lambda_{k}^{h_{l}}$ & $\eta\left(\lambda_{k, h_{l}}\right) / e\left(\lambda_{k, h_{l}}\right)$ & $\eta\left(\lambda_{k}^{h_{l}}\right) / e\left(\lambda_{k}^{h_{l}}\right)$ \\
\hline 1 & $\sqrt{2} / 10$ & 1 & $\sqrt{2} / 40$ & 2.619901684020 & 2.619902307450 & 0.16 & 0.16 \\
1 & $\sqrt{2} / 10$ & 2 & $\sqrt{2} / 80$ & 2.545994662814 & 2.545994677427 & 0.69 & 0.69 \\
1 & $\sqrt{2} / 10$ & 3 & $\sqrt{2} / 160$ & - & 2.468072524066 & - & 0.78 \\
2 & $\sqrt{2} / 10$ & 1 & $\sqrt{2} / 40$ & 3.540738971244 & 3.540738974231 & 5.75 & 5.75 \\
2 & $\sqrt{2} / 10$ & 2 & $\sqrt{2} / 80$ & 3.536561194304 & 3.536561194305 & 1.65 & 1.65 \\
2 & $\sqrt{2} / 10$ & 3 & $\sqrt{2} / 160$ & - & 3.534975905948 & - & 1.67 \\
3 & $\sqrt{2} / 10$ & 1 & $\sqrt{2} / 40$ & 9.869612668412 & 9.869612668412 & 244.51 & 244.51 \\
3 & $\sqrt{2} / 10$ & 2 & $\sqrt{2} / 80$ & 9.869604920374 & 9.869604920377 & 14.92 & 14.92 \\
3 & $\sqrt{2} / 10$ & 3 & $\sqrt{2} / 160$ & - & 9.869604433619 & - & 14.92 \\
5 & $\sqrt{2} / 10$ & 1 & $\sqrt{2} / 40$ & 11.392491049450 & 11.392491049225 & 7.11 & 7.11 \\
5 & $\sqrt{2} / 10$ & 2 & $\sqrt{2} / 80$ & 11.390607786437 & 11.390607786437 & 1.67 & 1.67 \\
5 & $\sqrt{2} / 10$ & 3 & $\sqrt{2} / 160$ & - & 11.389899921315 & - & 1.68 \\
\hline
\end{tabular}

Table 3: The results on the L-shaped domain by Scheme $1\left(P_{2}-P_{1}\right.$ element) for the eigenvalue problem of electric field $(r=0.95)$.

\begin{tabular}{cccccccc}
\hline$k$ & $H$ & $l$ & $h_{l}$ & $\lambda_{k, h_{l}}$ & $\lambda_{k}^{h_{l}}$ & $\eta\left(\lambda_{k, h_{l}}\right) / e\left(\lambda_{k, h_{l}}\right)$ & $\eta\left(\lambda_{k}^{h_{l}}\right) / e\left(\lambda_{k}^{h_{l}}\right)$ \\
\hline 1 & $\sqrt{2} / 10$ & 1 & $\sqrt{2} / 40$ & 1.550099678021 & 1.550100277590 & 1.88 & 1.88 \\
1 & $\sqrt{2} / 10$ & 2 & $\sqrt{2} / 80$ & 1.512784318422 & 1.512784324775 & 1.00 & 1.00 \\
1 & $\sqrt{2} / 10$ & 3 & $\sqrt{2} / 160$ & - & 1.492972425344 & - & 1.14 \\
2 & $\sqrt{2} / 10$ & 1 & $\sqrt{2} / 40$ & 3.534598663496 & 3.534598663588 & 14.65 & 14.65 \\
2 & $\sqrt{2} / 10$ & 2 & $\sqrt{2} / 80$ & 3.534154160497 & 3.534154160495 & 3.62 & 3.62 \\
2 & $\sqrt{2} / 10$ & 3 & $\sqrt{2} / 160$ & - & 3.534055109225 & - & 4.17 \\
3 & $\sqrt{2} / 10$ & 1 & $\sqrt{2} / 40$ & 9.869612641692 & 9.869612641692 & 243.23 & 243.23 \\
3 & $\sqrt{2} / 10$ & 2 & $\sqrt{2} / 80$ & 9.869604919289 & 9.869604919288 & 14.90 & 14.90 \\
3 & $\sqrt{2} / 10$ & 3 & $\sqrt{2} / 160$ & - & 9.869604433575 & - & 14.92 \\
5 & $\sqrt{2} / 10$ & 1 & $\sqrt{2} / 40$ & 11.389671749018 & 11.389671749041 & 28.83 & 28.83 \\
5 & $\sqrt{2} / 10$ & 2 & $\sqrt{2} / 80$ & 11.389519405478 & 11.389519405477 & 3.81 & 3.81 \\
5 & $\sqrt{2} / 10$ & 3 & $\sqrt{2} / 160$ & - & 11.389487122250 & - & 4.18 \\
\hline
\end{tabular}


In Tables $1-3, \lambda_{1, h_{l}}, \lambda_{2, h_{l}}, \ldots, \lambda_{5, h_{l}}$ denote the first five eigenvalues obtained by using the mixed element on $K^{h_{l}}$ directly; $\lambda_{1}^{h_{l}}, \lambda_{2}^{h_{l}}, \ldots, \lambda_{5}^{h_{l}}$ denote the first five eigenvalues obtained by Scheme 1:

$$
\begin{gathered}
e\left(\lambda_{k, h_{l}}\right)=\left|\lambda_{k, h_{l}}-\lambda_{k}\right|, \quad e\left(\lambda_{k}^{h_{l}}\right)=\left|\lambda_{k}^{h_{l}}-\lambda_{k}\right|, \\
\eta\left(\lambda_{k, h_{l}}\right)=\theta\left|\lambda_{k, h_{l}}-\lambda_{k, h_{l-1}}\right|, \quad \eta\left(\lambda_{k}^{h_{l}}\right)=\theta\left|\lambda_{k}^{h_{l}}-\lambda_{k}^{h_{l-1}}\right|, \quad(\theta=1) .
\end{gathered}
$$

From Tables 1-3, we can see that (1) $\lambda_{k}^{h_{l}}$ and $\lambda_{k, h_{l}}$ have the same accuracy. (2) It fails to find $\lambda_{k, h_{l}}$ by direct computation by using the mixed element on $K^{h_{l}}$ with $h_{l}=\sqrt{2} / 128$ in the case of the square domain and $h_{l}=\sqrt{2} / 160$ in the case of the L-shaped domain (here Matlab shows that Sparse lu with 4 outputs (UMFPACK) failed), but Scheme 1 still works. (3) $\eta\left(\lambda_{k}^{h_{l}}\right)=\left|\lambda_{k}^{h_{l}}-\lambda_{k}^{h_{l-1}}\right|$ is an efficient and reliable error indicator of $\lambda_{k}^{h_{l}}$.

It can be seen from Tables 1-3 that, in the calculation of error indicators, $\theta$ should be selected as follows: in the case of the square domain, $\theta=1 / 15$; in the case of the L-shaped domain, $\theta$ is equal to $1,3 / 5,1 / 15$, and $3 / 5$, respectively when $r=0.5$, and $\theta$ is equal to $1,1 / 4,1 / 15$ and $1 / 4$ respectively, when $r=0.95$.

Remark 5.1. Taking Table 1 , for example, we illustrate how to select $t_{i}$ next. In Table $1, h_{0}=$ $\sqrt{2} / 8, h_{1}=\sqrt{2} / 32, h_{2}=\sqrt{2} / 64$, and $h_{3}=\sqrt{2} / 128$. According to $h_{i}=h_{i-1}^{t_{i}}$, by calculation, we get that $t_{1} \approx 1.80, t_{2} \approx 1.22$, and $t_{3} \approx 1.18$.

\section{Acknowledgments}

The authors cordially thank the editor and the referees for their valuable comments which lead to the great improvement of this paper. This work is supported by National Natural Science Foundation of China (Grant no. 11161012).

\section{References}

[1] D. Boffi, P. Fernandes, L. Gastaldi, and I. Perugia, “Computational models of electromagnetic resonators: analysis of edge element approximation," SIAM Journal on Numerical Analysis, vol. 36, no. 4, pp. 1264-1290, 1999.

[2] A. Buffa and I. Perugia, "Discontinuous Galerkin approximation of the Maxwell eigenproblem," SIAM Journal on Numerical Analysis, vol. 44, no. 5, pp. 2198-2226, 2006.

[3] A. Buffa, P. Ciarlet Jr., and E. Jamelot, "Solving electromagnetic eigenvalue problems in polyhedral domains with nodal finite elements," Numerische Mathematik, vol. 113, no. 4, pp. 497-518, 2009.

[4] P. Ciarlet Jr. and G. Hechme, "Computing electromagnetic eigenmodes with continuous Galerkin approximations," Computer Methods in Applied Mechanics and Engineering, vol. 198, no. 2, pp. 358-365, 2008.

[5] S. Caorsi, P. Fernandes, and M. Raffetto, "On the convergence of Galerkin finite element approximations of electromagnetic eigenproblems," SIAM Journal on Numerical Analysis, vol. 38, no. 2, pp. 580-607, 2000.

[6] F. Kikuchi, "Mixed and penalty formulations for finite element analysis of an eigenvalue problem in electromagnetism," Computer Methods in Applied Mechanics and Engineering, vol. 64, pp. 509-521, 1987.

[7] Y. Yang, W. Jiang, Y. Zhang, W. Wang, and H. Bi, "A two-scale discretization scheme for mixed variational formulation of eigenvalue problems," Abstract and Applied Analysis, vol. 2012, Article ID 812914, 29 pages, 2012. 
[8] Y. Yang and H. Bi, "Two-grid finite element discretization schemes based on shifted-inverse power method for elliptic eigenvalue problems," SIAM Journal on Numerical Analysis, vol. 49, no. 4, pp. 16021624, 2011.

[9] H. Bi and Y. Yang, "Multi-scale discretizaiton scheme based on the Rayleigh quotient iterative method for the Steklov eigenvalue problem," Mathematical Problems in Engineering, vol. 2012, Article ID 487207, 18 pages, 2012.

[10] L. N. Trefethen and D. Bau III, Numerical Linear Algebra, SIAM, Philadelphia, Pa, USA, 1997.

[11] M. Costabel and M. Dauge, "Weighted regularization of Maxwell equations in polyhedral domains. A rehabilitation of nodal finite elements," Numerische Mathematik, vol. 93, no. 2, pp. 239-277, 2002.

[12] Y. Yang, Finite Element Methods for Eigenvalue Problems, Science Press, Beijing, China, 2012.

[13] D. Boffi, F. Brezzi, and L. Gastaldi, "On the convergence of eigenvalues for mixed formulations," Annali della Scuola Normale Superiore di Pisa IV, vol. 25, no. 1-2, pp. 131-154, 1997.

[14] F. Brezzi and M. Fortin, Mixed and Hybrid Finite Element Methods, vol. 15, Springer, New York, NY, USA, 1991.

[15] I. Babuška and J. Osborn, "Eigenvalue problems," in Finite Element Methods(Part 1), Handbook of Numerical Analysis, P. G. Ciarlet and J. L. Lions, Eds., vol. 2, pp. 641-787, Elsevier Science Publishers, North-Holand, 1991.

[16] B. Mercier, J. Osborn, J. Rappaz, and P.-A. Raviart, “Eigenvalue approximation by mixed and hybrid methods," Mathematics of Computation, vol. 36, no. 154, pp. 427-453, 1981.

[17] F. Chatelin, Spectral Approximation of Linear Operators, Academic Press, New York, NY, USA, 1983.

[18] H. Chen, S. Jia, and H. Xie, "Postprocessing and higher order convergence for the mixed finite element approximations of the Stokes eigenvalue problems," Applications of Mathematics, vol. 54, no. 3, pp. 237-250, 2009.

[19] P. G. Ciarlet, "Basic error estimates for elliptic problems," in Finite Element Methods (Part1), Handbook of Numerical Analysis, P. G. Ciarlet and J. L. Lions, Eds., vol. 2, pp. 21-343, Elsevier Science Publishers, North-Holand, 1991.

[20] X. Dai, J. Xu, and A. Zhou, "Convergence and optimal complexity of adaptive finite element eigenvalue computations," Numerische Mathematik, vol. 110, no. 3, pp. 313-355, 2008.

[21] V. Heuveline and R. Rannacher, "A posteriori error control for finite approximations of elliptic eigenvalue problems," Advances in Computational Mathematics, vol. 15, no. 1-4, pp. 107-138, 2001.

[22] D. Mao, L. Shen, and A. Zhou, "Adaptive finite element algorithms for eigenvalue problems based on local averaging type a posteriori error estimates," Advances in Computational Mathematics, vol. 25, no. 1-3, pp. 135-160, 2006.

[23] C. Amrouche, C. Bernardi, M. Dauge, and V. Girault, "Vector potentials in three-dimensional nonsmooth domains," Mathematical Methods in the Applied Sciences, vol. 21, no. 9, pp. 823-864, 1998.

[24] M. Costabel, "A coercive bilinear form for Maxwell's equations," Journal of Mathematical Analysis and Applications, vol. 157, no. 2, pp. 527-541, 1991.

[25] P. Ciarlet Jr., "Augmented formulations for solving Maxwell equations," Computer Methods in Applied Mechanics and Engineering, vol. 194, no. 2-5, pp. 559-586, 2005.

[26] P. Ciarlet Jr. and V. Girault, "inf-sup condition for the 3D, $P_{2}$-iso- $P_{1}$, Taylor-Hood finite element application to Maxwell equations," Comptes Rendus Mathématique, vol. 335, no. 10, pp. 827-832, 2002. 


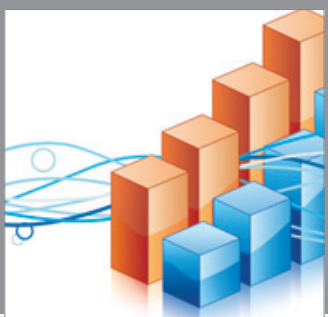

Advances in

Operations Research

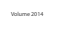

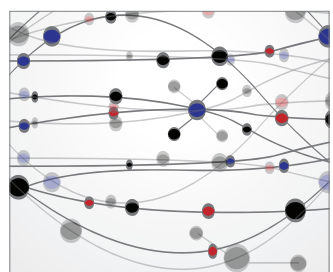

\section{The Scientific} World Journal
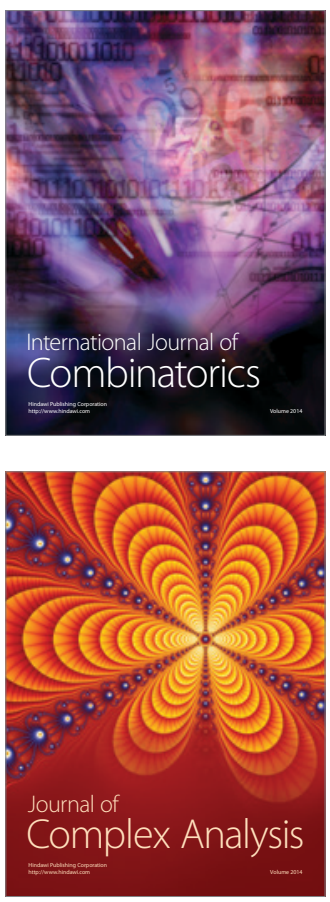

International Journal of

Mathematics and

Mathematical

Sciences
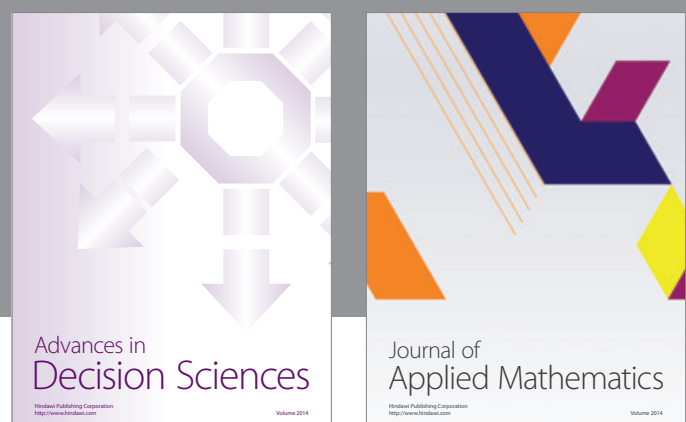

Journal of

Applied Mathematics
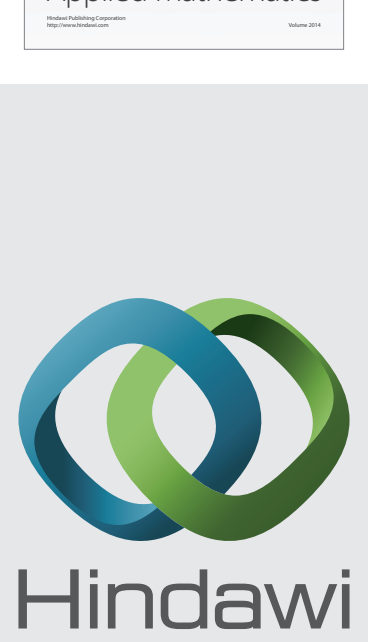

Submit your manuscripts at http://www.hindawi.com
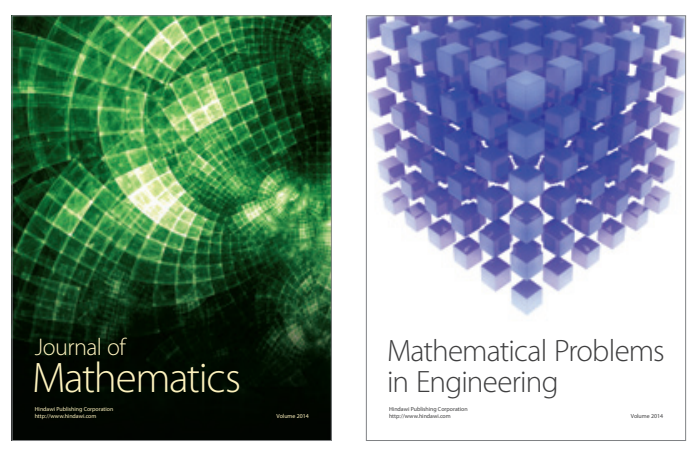

Mathematical Problems in Engineering
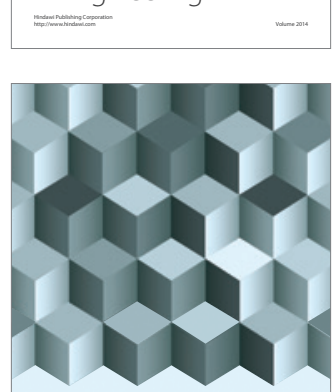

Journal of

Function Spaces
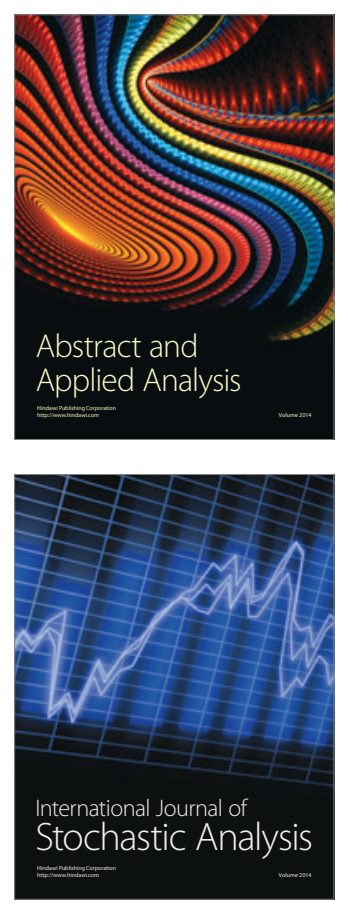

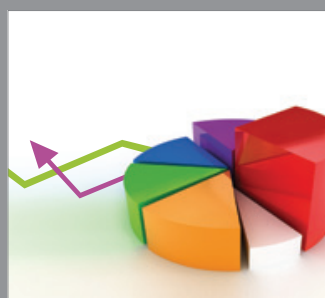

ournal of

Probability and Statistics

Promensencen
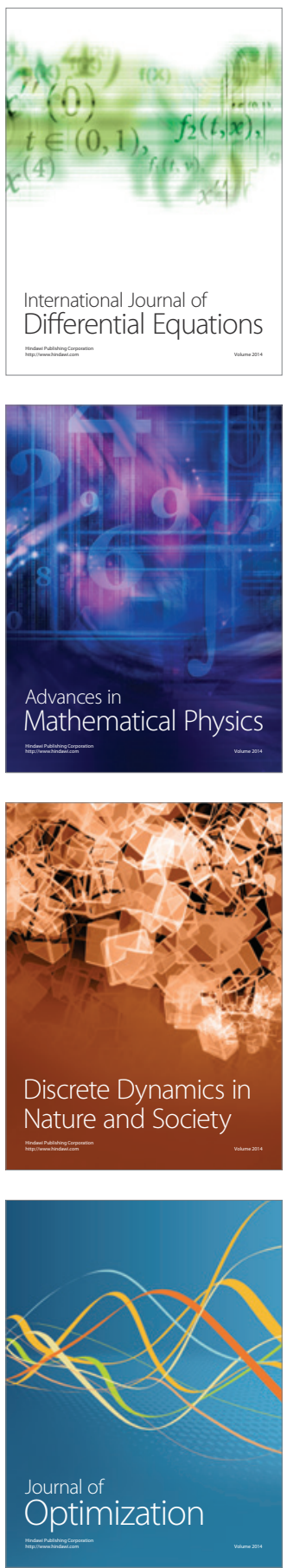